\title{
ANALYSIS AND OUTPUT TRACKING DESIGN FOR THE DIRECT CONTACT MEMBRANE DISTILLATION SYSTEM
}

\author{
MOHAMED GHATTASSI *, TAOUS-MERIEM LALEG ${ }^{\dagger}$, AND JEAN-CLAUDE VIVALDA ${ }^{\dagger}$
}

\begin{abstract}
This paper discusses the mathematical properties of a recently developed mathematical model of a direct contact membrane distillation system. The model consists of two-dimensional advection diffusion system coupled at the boundary. A semi-group framework is used to analyze the model. First, the infinitesimal generator operator and its properties are studied. Then, existence and uniqueness of the solutions are investigated using the theory of operators. Some regularity results of the solution are also established. A particular case showing the diagonal property of the principal operator is studied. However, based on this new partial differential model we formulated our problem of output tracking design for the parabolic distillation system. Using a partial boundary measurement, we first propose an extended state observer to estimate both system state and the disturbance. Then we design a servomechanism and thereafter an output feedback controller. Thus by some regularity for the reference signal and the disturbance vanish, we prove the exponential decay of the output tracking error. Moreover, we show the performance of the control strategy in presence of the measurement noise.
\end{abstract}

Key words. Direct Contact Membrane Distillation System, Well-Posedness Criteria, disturbance rejection, disturbance rejection control.

1. Introduction. The access to drinking water is getting more and more problematic as a result of the limited natural freshwater resources. The potable water demand is increasing due to the rapid population growth and to impacts of climate change. As a consequence, many countries rely on desalination to address their potable water demand. Indeed, desalination has been recognized as one of the most promising approaches to reduce water shortage in arid regions through the production of fresh water from seawater and saline groundwater. Among conventional yet innovative water desalination technologies is membrane distillation (MD). MD has great potential for sustainable high quality water supply. It is a low energy and effective method for water treatment and desalination. It consists of a separation process driven by temperature gradient, where hot salt water is circulated in one side (the feed side) of a hydrophobic porous membrane, while cold-fresh stream is circulated in the other side (the permeate side), thus creating a difference of partial pressure between the two sides of the membrane that constitutes the main driving force of the process. There are different configurations for MD system such as Direct Contact Membrane Distillation (DCMD), Air Gap Membrane Distillation (AGMD), Sweeping Gas Membrane Distillation (SGMD) and Vacuum Membrane Distillation (VMD). For more details about the MD technology and its configurations, the reader can refer to $[16,17,6]$ and references therein. Many studies have been conducted recently by engineers to propose accurate mathematical models of MD systems and to develop efficient modelbased control and monitoring strategies [15, 24, 22]. An accurate mathematical model will also allow the optimization of the system to increase its efficiency. Among the proposed MD models is a system of two dimensional advection diffusion equations coupled at the boundary. This dynamical model has been proposed for the DCMD configuration and has been validated experimentally in [7].

\footnotetext{
${ }^{*}$ Computer, Electrical, and Mathematical Sciences and Engineering Division, King Abdullah University of Science and Technology (KAUST), Thuwal, 53955-6900, Kingdom of Saudi Arabia, (mohamed.ghattassi@kaust.edu.sa,taousmeriem.laleg@kaust.edu.sa )

${ }^{\dagger}$ J.-C. Vivalda is with Inria, Villers-lès-Nancy, F-54600, France- Université de Lorraine, IECL, UMR 7502, Vandœuvre-lès-Nancy, F-54506, France - CNRS, IECL,UMR 7502, Vandœuvre-lèsNancy, F-54506, France, Jean-Claude.Vivalda@inria.fr
} 
The aim of this paper is to study and analyze the mathematical properties of the DCMD model describing heat transfer in the DCMD process and given by a two dimensional advection diffusion parabolic system coupled at the boundary. The framework of semigroup theory has been considered. Using some classical arguments for the analysis of partial differential equations (PDEs), the model operator is shown to be m-dissipative. Moreover, it is proven that for any initial conditions, the solution of the system tends to an equilibrium as time tends to infinity. In particular, we will show that this operator is diagonal in the co-current configuration, if some additional conditions on thermal conductivity and flow rate are satisfied. It is worth to point our that systems of advection-diffusion equations represent an important class of PDEs that arise in many problems of science and engineering. In this context, there exists some papers that have been devoted to the study of the reaction-advection-diffusion systems for linear and nonlinear cases, see [3, 21, 4] and references therein. The authors study the well-posedness and the blow-up of the solution for a class of nonlinear reaction-advection-diffusion system. The systems studied in these work present an internal coupling. On the other hand based on this new model, we will develop an output feedback to track the temperature of the 2D parabolic system using some boundary measurements. This kind of system will be treated for the first time in our work. However, there are some techniques used for the control design problem of the parabolic systems in the multidimensional case. Among the backstepping technique, we refer the reader to the pioneering work of Thomas Meurer and and his collaborators $[19,20]$. It should be noted that in the beginning, we started to see this control problem based on this method but, in our model this technique presents some limitations, because our operator is self-adjoint only for some particular values of thermal conductivity and flow rate coefficients suddenly, we are oriented towards the Active disturbance rejection control (ADRC) method. This new technique needs only an analytic semigroup operator. Moreover, ADRC a powerful solution proposed to deal with control problems for systems subject to external disturbances. The main idea is to provide an effective estimation of the disturbance, which is incorporated in the control law to allow an efficient decoupling of the actual disturbance. The topic has been well covered in finite dimensional systems, for example, [11, 5]. However, there are also some studies that investigate the ADRC for PDEs. For example, this technique has been proposed for wave equation in $[12,13]$. Then we explored it for parabolic equations by Bao-Zhu Guo [10, 14, 27]. In [10] an unknown input type state observer has been proposed to stabilize an unstable one dimensional heat equation with external disturbance and boundary uncertainty, which has been combined to a backstepping control. In [14], the authors considered the boundary output tracking for a one dimensional heat equation with external disturbance. A design of an observer to estimate the disturbance is proposed and a servo system which consists of an output feedback boundary control law has been derived. Recently, in [27], the authors extended the performance output tracking for a boundary controlled multidimensional heat equation with external disturbance and unknown internal nonlinear uncertainty. In this work, we propose an extension of this method for the system of parabolic equation weakly coupled at the boundary by one side control in order to track the temperature at the output of the feed and the permeate, respectively.

This paper is organized as follows. In section 2 we describe a mathematical model for the heat transfer in the DCMD systems. Section 3 formulates the problem using the framework of operator theory; where we prove that the operator related to the DCMD system is m-dissipative. The proof of the existence and uniqueness for the solution of the DCMD elliptic system is established in section 4. In section 5 the co- 
current DCMD case is presented and it is shown that under some additional conditions the operator is diagonal. Moreover, in section 6, we formulate the output tracking problem for the DCMD system based on the ADRC technique. Finally, in section 7 we address some natural open questions arising after our study.

2. Mathematical modeling of heat transfer in DCMD process. The model geometry consists of a feed inlet boundary $\mathrm{B}_{1}$, feed outlet boundary $\mathrm{B}_{3}$, permeate inlet boundary $\mathrm{B}_{4}$, permeate outlet boundary $\mathrm{B}_{6}$. In this module, the vapor generated in the feed solution (warm sea water) is forced to pass through the membrane dry pores to the permeate side (cold water), following thermodynamics rules. Hereafter, we outline the equations describing the evolutions of the temperatures in the feed and permeate rooms of the devices, more details can be found in [7] or [24].

We denote by $f(t, x, y)$ the temperature of the warm water and by $p(t, x, y)$ the temperature of the cold water at time $t$ and at the point of coordinates $(x, y)$; we denote also by $\Omega_{f}$ and $\Omega_{p}$ the rectangles $[0, \ell] \times[0, L]$ and $\left[\delta_{m}+\ell, \delta_{m}+2 \ell\right] \times[0, L]$ respectively (here $\delta_{m}$ denotes the membrane thickness, see Fig. 1).

The mathematical model for the evolution of the temperatures in the device involves a diffusion and a convection term. The equations write, see [24]

$$
\begin{aligned}
& \partial_{t} f(t, x, y)=\alpha_{f} \Delta f(t, x, y)-\beta_{f} \partial_{y} f(t, x, y) \quad \text { for } t \geq 0 \text { and }(x, y) \in \Omega_{f} \\
& \partial_{t} p(t, x, y)=\alpha_{p} \Delta p(t, x, y)-\beta_{p} \partial_{y} p(t, x, y) \quad \text { for } t \geq 0 \text { and }(x, y) \in \Omega_{p} .
\end{aligned}
$$

The coefficients $\alpha_{f}, \beta_{f}$ and $\alpha_{p}$ are positive and are assumed to be constant, they depend on the thermal conductivity and on the densities of the fluids (see [24] ); specifically they are defined as follows

$$
\alpha_{f}=\frac{\kappa_{f}}{\rho_{f} C_{f}}, \quad \alpha_{p}=\frac{\kappa_{p}}{\rho_{p} C_{p}} .
$$

Here $\kappa_{k}, \rho_{k}$ and $C_{k},(k \in\{p, f\})$ denote respectively the thermal conductivity of fluid, liquid density of fluid and specific heat capacity of fluid. The coefficients $\beta_{f}>0$ and $\beta_{p}$ denote the velocities of the flow in the feed and permeate side respectively. The velocity $\beta_{p}$ in the permeate is negative in the counter-current case, Fig. 1 (a) and positive for the co-current presentation, Fig. 1 (b). The boundary conditions are a mix of Dirichlet, von Neumann and Robin conditions, they are:

On the boundary $\mathrm{B}_{1}$.

$$
f(t, x, 0)=T_{f} \quad \text { for every } 0 \leq x \leq \ell
$$

On the boundary $\mathrm{B}_{2}$.

$$
\partial_{x} f(t, 0, y)=0 \quad \text { for every } 0 \leq y \leq L
$$

On the boundary $\mathrm{B}_{3}$.

$$
\partial_{y} f(t, x, L)=0 \quad \text { for every } 0 \leq x \leq \ell
$$

On the boundary $\mathrm{B}_{4}$.

$$
\begin{array}{cll}
p(t, x, L)=T_{p} & \text { for every } \ell+\delta_{m} \leq x \leq 2 \ell+\delta_{m}, & \text { Fig. } 1 \text { (a) } \\
p(t, x, 0)=T_{p} & \text { for every } \ell+\delta_{m} \leq x \leq 2 \ell+\delta_{m}, & \text { Fig. } 1 \text { (b) }
\end{array}
$$

On the boundary $\mathrm{B}_{5}$.

$$
\partial_{x} p\left(t, 2 \ell+\delta_{m}, y\right)=0 \quad \text { for every } 0 \leq y \leq L
$$




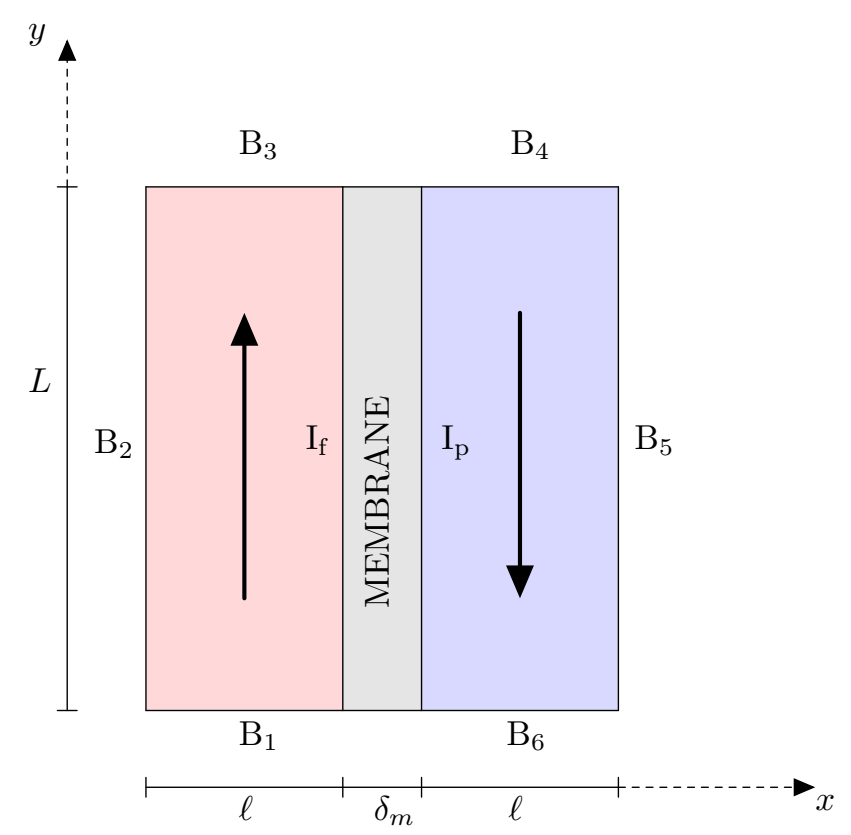

(a) Counter-current presentation

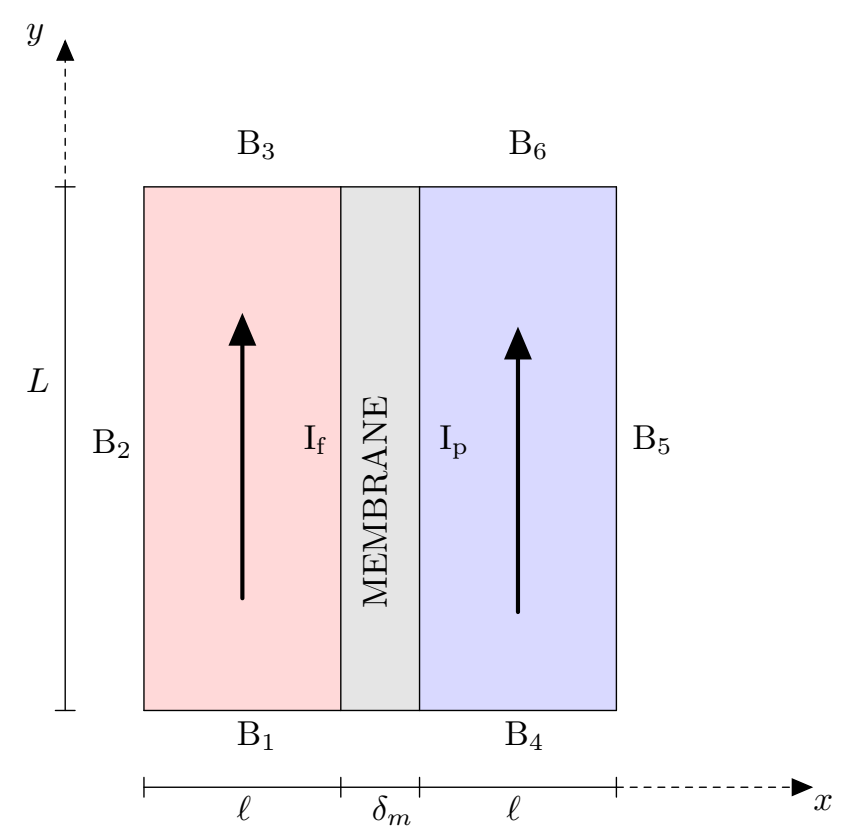

(b) Co-current presentation

Figure 1. Schematic of the device

On the boundary $\mathrm{B}_{6}$.

$$
\begin{array}{ll}
\partial_{y} p(t, x, 0)=0 & \text { for every } \ell+\delta_{m} \leq x \leq 2 \ell+\delta_{m} \text { Fig. } 1 \text { (a) } \\
\partial_{y} p(t, x, L)=0 & \text { for every } \ell_{4}+\delta_{m} \leq x \leq 2 \ell+\delta_{m}, \text { Fig. } 1 \text { (b) }
\end{array}
$$




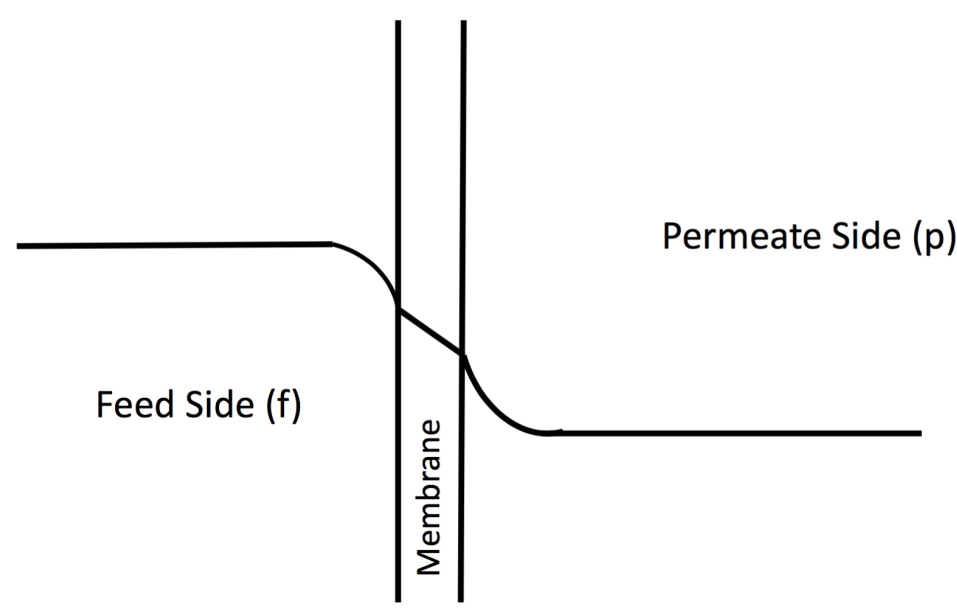

FIGURE 2. Temperature evolution from feed $(f)$ to permeate (p) in DCMD

On the interfaces $\mathrm{I}_{f}$ ane $\mathrm{I}_{p}$.

$$
\begin{aligned}
& k_{f} \partial_{x} f(t, \ell, y)=-\left(J \lambda+\frac{k_{m}}{\delta_{m}} f(t, \ell, y)-\frac{k_{m}}{\delta_{m}} p\left(t, \ell+\delta_{m}, y\right)\right) \\
& k_{p} \partial_{x} p(t, \ell, y)=-\left(J \lambda+\frac{k_{m}}{\delta_{m}} f(t, \ell, y)-\frac{k_{m}}{\delta_{m}} p\left(t, \ell+\delta_{m}, y\right)\right)
\end{aligned}
$$

The surface temperature on the feed side of the membrane equals the feed temperature $f$ and the surface temperature on the permeate side of the membrane equals the bulk temperature $p$ of the condensing fluid. Nevertheless, the process is known to suffer from temperature polarization as depicted in figure 2 causing a decrease in permeate fluxes [1].

The term $J$ denotes the permeate flux through the membrane; the mass transport mechanism in the membrane pores is governed by three basic mechanisms known as: Knudsen diffusion, molecular diffusion and Poiseuille flow, the reader is referred to $[22,24]$ and $[16$, Chapter 10] for more details. The term $\lambda$ is the latent heat of water, these terms depend on the temperature; the product $J \lambda$ is very small (about $10^{-6}$ ) and will be neglected in the sequel. the terms $k_{f}, k_{p}$ and $k_{m}$ are thermal conductivity coefficients.

As this model does not take into account the physical phenomena inside the membrane, we shall rewrite it in such a way that the two unknown functions $f$ and $p$ are defined on the same domain. To this end, one make the following change of unknown function: $\tilde{p}(t, x, y)=p\left(t, 2 \ell+\delta_{m}-x, y\right)$ where $(x, y) \in[0, \ell] \times[0, L]$. Hereafter, the partial differential equations as well as the boundary conditions are rewritten with the unknown functions $f$ and $\tilde{p}$. For the sake, of readability, we keep the notation $p(t, x, y)$ (instead of $\tilde{p}$ ), moreover, without loss of generality, we assume that $\ell=1$. The rest of the paper will examine the counter current case. However, the findings remain true for the co-current process. The domain of definition of the PDE's is $\Omega:=(0,1) \times(0, L)$, the considered advection diffusion system writes 


$$
\left\{\begin{array}{lr}
\partial_{t} f(t, x, y)-\alpha_{f} \Delta f(t, x, y)+\beta_{f} \partial_{y} f(t, x, y)=0, & t \geq 0,(x, y) \in \Omega \\
\partial_{t} p(t, x, y)-\alpha_{p} \Delta p(t, x, y)-\beta_{p} \partial_{y} p(t, x, y)=0 & t \geq 0,(x, y) \in \Omega \\
f(t, x, 0)=T_{f} & t \geq 0,0 \leq x \leq 1, \\
\partial_{x} f(t, 0, y)=0 & t \geq 0,0 \leq y \leq L, \\
\partial_{y} f(t, x, L)=0 & t \geq 0,0 \leq x \leq 1, \\
p(t, x, L)=T_{p} & t \geq 0,0 \leq x \leq 1, \\
\partial_{x} p(t, 0, y)=0 & t \geq 0,0 \leq y \leq L, \\
\partial_{y} p(t, x, 0)=0 & t \geq 0,0 \leq x \leq 1, \\
\partial_{x} f(t, 1, y)=-\gamma_{f}(f(t, 1, y)-p(t, 1, y)) & t \geq 0,0 \leq y \leq L, \\
\partial_{x} p(t, 1, y)=\gamma_{p}(f(t, 1, y)-p(t, 1, y)) & t \geq 0,0 \leq y \leq L, \\
f(0, x, y)=f_{0}(x, y) & (x, y) \in \Omega, \\
p(0, x, y)=p_{0}(x, y) & (x, y) \in \Omega .
\end{array}\right.
$$

Here the constants $\gamma_{f}$ and $\gamma_{p}$ are respectively equal to $k_{m}\left(\delta_{m} k_{f}\right)^{-1}$ and $k_{m}\left(\delta_{m} k_{p}\right)^{-1}$. $T_{f}, T_{p}, f_{0}$ and $p_{0}$ are the initial data of the system.

REMARK. A simplification of the DCMD model has been proposed in [7] under appropriate physical assumptions. Indeed, the vertical thermal diffusivity for the considered geometry has been neglected, the width $\ell$ being sufficiently small compared to the length L. This assumption is based on the fact that the horizontal diffusivity is dominant.

$$
\left\{\begin{array}{lr}
\partial_{t} f(t, x, y)-\alpha_{f} \partial_{x x} f(t, x, y)+\beta_{f} \partial_{y} f(t, x, y)=0, & t \geq 0,(x, y) \in \Omega \\
\partial_{t} p(t, x, y)-\alpha_{p} \partial_{x x} p(t, x, y)-\beta_{p} \partial_{y} p(t, x, y)=0 & t \geq 0,(x, y) \in \Omega \\
f(t, x, 0)=T_{f} & t \geq 0,0 \leq x \leq 1, \\
\partial_{x} f(t, 0, y)=0 & t \geq 0,0 \leq y \leq L, \\
p(t, x, L)=T_{p} & t \geq 0,0 \leq x \leq 1, \\
\partial_{x} p(t, 0, y)=0 & t \geq 0,0 \leq y \leq L, \\
\partial_{x} f(t, 1, y)=-\gamma_{f}(f(t, 1, y)-p(t, 1, y)) & t \geq 0,0 \leq y \leq L, \\
\partial_{x} p(t, 1, y)=\gamma_{p}(f(t, 1, y)-p(t, 1, y)) & t \geq 0,0 \leq y \leq L, \\
f(0, x, y)=f_{0}(x, y) & (x, y) \in \Omega, \\
p(0, x, y)=p_{0}(x, y) & (x, y) \in \Omega .
\end{array}\right.
$$

To study the advection diffusion system (11), we shall place ourselves in the framework of operators theory.

3. The operator related to the membrane distillation system. First, we introduce the domain of the operator: consider the space $\mathbf{E}$ which is the set of pairs $(f, p)$ in $\left[H^{1}(\Omega) \cap C^{1}(\bar{\Omega})\right]^{2}$ such that

- $f(x, 0)=p(x, L)=0$ for every $x \in(0,1)$;

- $\partial_{x} f(0, y)=\partial_{x} p(0, y)=0$, for every $y \in(0, L)$;

- $\partial_{y} f(x, L)=\partial_{y} p(x, 0)=0$, for every $x \in(0,1)$;

- $\partial_{x} f(1, y)=-\gamma_{f}(f(1, y)-p(1, y))$, for every $y \in(0, L)$;

- $\partial_{x} p(1, y)=\gamma_{p}(f(1, y)-p(1, y))$ for every $y \in(0, L)$. 
The space $H^{1}(\Omega) \times H^{1}(\Omega)$ is equipped with the product topology, so the norm of an element $(f, p) \in H^{1}(\Omega) \times H^{1}(\Omega)$ is defined by

$$
\|(f, p)\|^{2}=\int_{\Omega} f^{2}+\int_{\Omega}|\nabla f|^{2}+\int_{\Omega} p^{2}+\int_{\Omega}|\nabla p|^{2} .
$$

On $L^{2}(\Omega) \times L^{2}(\Omega)$, we consider the following inner product:

$$
\langle(f, p),(g, q)\rangle:=\alpha_{p} \gamma_{p} \int_{\Omega} f(x, y) g(x, y) \mathrm{d} x \mathrm{~d} y+\alpha_{f} \gamma_{f} \int_{\Omega} p(x, y) q(x, y) \mathrm{d} x \mathrm{~d} y
$$

notice that this inner product induces the product topology on $L^{2}(\Omega) \times L^{2}(\Omega)$. We denote by $\mathcal{H}_{\mathrm{bc}}^{1}$ the closure of $\mathbf{E}$ in $\left[H^{1}(\Omega)\right]^{2}$; from the Poincaré's inequality, the induced norm on $\mathcal{H}_{\mathrm{bc}}^{1}$ defined by (12) is equivalent to the following one

$$
\|(f, p)\|_{\mathcal{H}_{\mathrm{bc}}^{1}}^{2}=\alpha_{f} \int_{\Omega}\|\nabla f\|^{2}+\alpha_{p} \int_{\Omega}\|\nabla p\|^{2}, \quad(f, p) \in \mathcal{H}_{\mathrm{bc}}^{1} .
$$

We then denote by $A_{0}$ the operator whose domain is given by

$$
\mathcal{D}\left(A_{0}\right):=\left\{(f, p) \in \mathcal{H}_{\mathrm{bc}}^{1} \mid(\Delta f, \Delta p) \in\left[L^{2}(\Omega)\right]^{2}\right\}
$$

and which is defined by

$$
A_{0}(f, p)=\left(\alpha_{f} \Delta f, \alpha_{p} \Delta p\right) .
$$

We introduce also the operator $B_{0}$, whose domain is the one of $A_{0}$ and which is defined by

$$
B_{0}(f, p)=\left(-\beta_{f} \partial_{y} f, \beta_{p} \partial_{y} p\right) ;
$$

finally we define operator $A$, related to system (11) by

$$
A=A_{0}+B_{0} .
$$

3.1. The operator $A_{0}$. In this section, we shall prove that the operator $A_{0}$ is, as the laplacian operator, self adjoint and m-dissipative. The proof is analogous to the one which shows these classical properties of the laplacian operator (see e.g. [25]). We begin by the following proposition.

Proposition 1. The embedding operator from $\mathcal{H}_{\mathrm{bc}}^{1}$ to $\left[L^{2}(\Omega)\right]^{2}$ is compact.

Proof. We denote by $J$ the embedding $\mathcal{H}_{\mathrm{bc}}^{1} \hookrightarrow\left[L^{2}(\Omega)\right]^{2}$ From the elementary theory of Fourier series we know that the family $\left(\varphi_{\alpha}\right)_{\alpha \in \mathbb{N}^{2}}$ defined by

$$
\varphi_{\alpha}=\frac{2}{\sqrt{L}} \sin \left(\alpha_{1} \pi x\right) \sin \left(\frac{\alpha_{2} \pi}{L} y\right), \quad \alpha:=\left(\alpha_{1}, \alpha_{2}\right) \in \mathbb{N}^{2},
$$

is an orthonormal basis for $L^{2}(\Omega)$. In this proof, we need the notation $\|\alpha\|^{2}=\alpha_{1}^{2}+\alpha_{2}^{2}$ for a multi-index $\alpha \in \mathbb{N}^{2}$. Let $(f, p) \in \mathcal{H}_{\mathrm{bc}}^{1}$. Then

$$
\|f\|_{L^{2}(\Omega)}^{2}=\sum_{\alpha \in \mathbb{N}^{2}}\left|\left\langle f, \varphi_{\alpha}\right\rangle\right|^{2}
$$

and

$$
\|f\|_{H^{1}(\Omega)}^{2}=\sum_{\alpha \in \mathbb{N}^{2}}\left(1+\|\alpha\|^{2}\right)\left|\left\langle f, \varphi_{\alpha}\right\rangle\right|^{2} .
$$


From the above formulas it follows that if $m \in \mathbb{N}$, and if $J_{m} \in \mathcal{L}\left(\mathcal{H}_{\mathrm{bc}}^{1}, L^{2}(\Omega)\right)$ is defined by

$$
J_{m}(f, p)=\sum_{\alpha \in \mathbb{N}^{2},\|\alpha\|^{2} \leqslant m}\left\langle f, \varphi_{\alpha}\right\rangle \varphi_{\alpha}+\sum_{\alpha \in \mathbb{N}^{2},\|\alpha\|^{2} \leqslant m}\left\langle p, \varphi_{\alpha}\right\rangle \varphi_{\alpha},
$$

then, as

$$
\sum_{\alpha \in \mathbf{N}^{2}}\|\alpha\|^{2}\left|\left\langle f, \varphi_{\alpha}\right\rangle\right|^{2} \geq m \sum_{\substack{\alpha \in \mathbf{N}^{2} \\\|\alpha\|^{2}>m}}\left|\left\langle f, \varphi_{\alpha}\right\rangle\right|^{2} \text { and } \sum_{\alpha \in \mathbf{N}^{2}}\|\alpha\|^{2}\left|\left\langle p, \varphi_{\alpha}\right\rangle\right|^{2} \geq m \sum_{\substack{\alpha \in \mathbf{N}^{2} \\\|\alpha\|^{2}>m}}\left|\left\langle p, \varphi_{\alpha}\right\rangle\right|^{2}
$$

we have

$$
\left\|J(f, p)-J_{m}(f, p)\right\|_{L^{2}(\Omega)}^{2} \leqslant \frac{1}{1+m}\|(f, p)\|_{\mathcal{H}_{\mathrm{bc}}^{1}},
$$

and so

$$
\lim _{m \rightarrow \infty} J_{m}=J .
$$

Since the dimension of $\operatorname{Ran}\left(J_{m}\right)$ is finite, this implies that $J$ is compact (see [25, Proposition 12.2.2]).

Theorem 2. The operator $A_{0}$ is self-adjoint and diagonalizable.

Proof. Take $(f, p)$ and $(g, q)$ in $\mathcal{D}\left(A_{0}\right)$; integrating two times by parts, we obtain

$$
\begin{aligned}
& \int_{\Omega} \frac{\partial^{2} f}{\partial x^{2}}(x, y) g(x, y) \mathrm{d} x \mathrm{~d} y=\int_{0}^{L} \frac{\partial f}{\partial x}(1, y) g(1, y) \mathrm{d} y \\
&+-\int_{0}^{L} f(1, y) \frac{\partial g}{\partial x}(1, y) \mathrm{d} y \\
&+\int_{\Omega} f(x, y) \frac{\partial^{2} g}{\partial x^{2}}(x, y) \mathrm{d} x \mathrm{~d} y \\
&=\gamma_{f} \int_{0}^{L}(g(1, y) p(1, y)-f(1, y) q(1, y)) \mathrm{d} y \\
&+\int_{\Omega} f(x, y) \frac{\partial^{2} g}{\partial x^{2}}(x, y) \mathrm{d} x \mathrm{~d} y,
\end{aligned}
$$

and

$$
\int_{\Omega} \frac{\partial^{2} f}{\partial y^{2}}(x, y) g(x, y) \mathrm{d} x \mathrm{~d} y=\int_{\Omega} f(x, y) \frac{\partial^{2} g}{\partial y^{2}}(x, y) \mathrm{d} x \mathrm{~d} y
$$

Analogous computations lead to

$$
\begin{array}{r}
\int_{\Omega} \Delta p(x, y) q(x, y) \mathrm{d} x \mathrm{~d} y=\gamma_{p} \int_{0}^{L}(f(1, y) q(1, y)-g(1, y) p(1, y)) \mathrm{d} y \\
+\int_{\Omega} p(x, y) \Delta q(x, y) \mathrm{d} x \mathrm{~d} y .
\end{array}
$$


So we have

$$
\begin{gathered}
\left\langle A_{0}(f, p),(g, q)\right\rangle=\alpha_{p} \gamma_{p} \alpha_{f} \gamma_{f} \int_{0}^{L}(g(1, y) p(1, y)-f(1, y) q(1, y)) \mathrm{d} y \\
+\alpha_{p} \gamma_{p} \alpha_{f} \int_{\Omega} f(x, y) \Delta g(x, y) \mathrm{d} x \mathrm{~d} y \\
+\alpha_{f} \gamma_{f} \alpha_{p} \gamma_{p} \int_{0}^{L}(f(1, y) q(1, y)-g(1, y) p(1, y)) \mathrm{d} y \\
\quad+\alpha_{f} \gamma_{f} \alpha_{p} \int_{\Omega} p(x, y) \Delta q(x, y) \mathrm{d} x \mathrm{~d} y \\
=\alpha_{p} \gamma_{p} \int_{\Omega} f(x, y)\left(\alpha_{f} \Delta g(x, y)\right) \mathrm{d} x \mathrm{~d} y \\
\quad+\alpha_{f} \gamma_{f} \int_{\Omega} p(x, y)\left(\alpha_{p} \Delta q(x, y)\right) \mathrm{d} x \mathrm{~d} y \\
=\left\langle(f, p), A_{0}(g, q)\right\rangle,
\end{gathered}
$$

which shows that the operator $A_{0}$ is symmetric. We shall now show that $A_{0}$ is selfadjoint; to do this, it is enough to prove that $A_{0}$ is onto, the proof is almost the same as in [25, Proposition 3.2.4]. that

Take $(u, v) \in\left[L^{2}(\Omega)\right]^{2}$, we have to prove the existence of $(f, p)$ in $\mathcal{D}\left(A_{0}\right)$ such

$$
A_{0}(f, p)=(u, v) .
$$

First notice that the mapping

$$
(g, q) \longrightarrow \int_{\Omega} u g+\int_{\Omega} v q
$$

is a bounded linear functional on $\mathcal{H}_{\mathrm{bc}}^{1}$. By the Riesz representation theorem, there exists $(f, p) \in \mathcal{H}_{\mathrm{bc}}^{1}$ such that

$$
\langle(f, p),(g, q)\rangle_{\mathcal{H}_{\mathrm{bc}}^{1}}=\langle(u, v),(g, q)\rangle_{\left[L^{2}(\Omega)\right]^{2}}
$$

the inner product in the left-hand member of this equality being the one related to the norm defined by (14). Denoting by $\mathcal{D}(\Omega)$ the space of smooth functions with compact support in $\Omega$, we notice that, as $\mathcal{D}(\Omega) \times \mathcal{D}(\Omega) \subset \mathcal{H}_{\mathrm{bc}}^{1}$, the above equality can also be written for any $g, q \in \mathcal{D}(\Omega)$; so, in the sense of distributions, we have

$$
\begin{aligned}
\langle(f, p),(g, q)\rangle_{\mathcal{H}_{\mathrm{bc}}^{1}} & =\alpha_{f} \int_{\Omega}(\nabla f) \cdot(\nabla g) \mathrm{d} x \mathrm{~d} y+\alpha_{p} \int_{\Omega}(\nabla p) \cdot(\nabla q) \mathrm{d} x \mathrm{~d} y \\
& =-\alpha_{f}\langle\Delta f, g\rangle_{\mathcal{D}^{\prime}, \mathcal{D}}-\alpha_{p}\langle\Delta p, q\rangle_{\mathcal{D}^{\prime}, \mathcal{D}} .
\end{aligned}
$$

This equality is true for every $(g, q) \in[\mathcal{D}(\Omega)]^{2}$, so we have

$$
-\alpha_{f} \Delta f=u, \text { in } \mathcal{D}^{\prime}(\Omega), \quad-\alpha_{p} \Delta p=v, \text { in } \mathcal{D}^{\prime}(\Omega) .
$$

Since, $(u, v) \in\left[L^{2}(\Omega)\right]^{2}$, we obtain that

$$
\alpha_{f} \Delta f \in L^{2}(\Omega), \quad \alpha_{p} \Delta p \in L^{2}(\Omega) .
$$

Thus $(f, p) \in \mathcal{D}\left(A_{0}\right)$ and

$$
A_{0}(f, p)=(u, v),
$$


hence $A_{0}$ is onto and we can conclude that $A_{0}$ is a self adjoint.

Finally, according to Proposition 1, the embedding $J: \mathcal{H}_{\mathrm{bc}}^{1} \hookrightarrow\left[L^{2}(\Omega)\right]^{2}$ is compact, therefore $A_{0}^{-1}=J \circ A_{0}^{-1}$ is compact and hence, by Proposition [25, Theorem 3.2.12], $A_{0}$ is diagonalizable with an orthonormal basis $\left(\varphi_{k}, \psi_{k}\right)$ of eigenvectors and the corresponding sequence of eigenvalues $\left(\lambda_{k}\right)$ satisfies and $\lim _{|k| \rightarrow \infty} \lambda_{k}=\infty$.

3.2. The operator $A$. In this section we shall prove that $A$ is m-dissipative with respect to the inner product defined in (13); the proof is in the same spirit as the proof of [23, Theorem 3.2]. We shall see first that $A$ is dissipative.

Proposition 3. For every $t \in[0,1]$, the operator $A_{0}+t B_{0}$ is dissipative; moreover, the operator $A_{0}$ is m-dissipative.

Proof. Take $(f, p) \in \mathcal{D}\left(A_{0}\right)$, we compute first $\left\langle A_{0}(f, p),(f, p)\right\rangle$ : integrating by parts, we get

$$
\begin{aligned}
\int_{\Omega} \frac{\partial^{2} f}{\partial x^{2}} f & =\int_{0}^{L}\left[\frac{\partial f}{\partial x}(x, y) f(x, y)\right]_{x=0}^{x=1} \mathrm{~d} y-\int_{\Omega}\left(\frac{\partial f}{\partial x}\right)^{2} \mathrm{~d} x \mathrm{~d} y \\
& =-\gamma_{f} \int_{0}^{L} f(1, y)(f(1, y)-p(1, y)) \mathrm{d} y-\int_{\Omega}\left(\frac{\partial f}{\partial x}\right)^{2} \mathrm{~d} x \mathrm{~d} y
\end{aligned}
$$

On the other hand

$$
\begin{aligned}
\int_{\Omega} \frac{\partial^{2} f}{\partial y^{2}} f & =\int_{0}^{1}\left[\frac{\partial f}{\partial y}(x, y) f(x, y)\right]_{y=0}^{y=1} \mathrm{~d} x-\int_{\Omega}\left(\frac{\partial f}{\partial y}\right)^{2} \mathrm{~d} x \mathrm{~d} y \\
& =-\int_{\Omega}\left(\frac{\partial f}{\partial x}\right)^{2} \mathrm{~d} x \mathrm{~d} y
\end{aligned}
$$

An analogous computation leads to

$$
\int_{\Omega}(\Delta p) p \mathrm{~d} x \mathrm{~d} y=\gamma_{p} \int_{0}^{L} p(1, y)(f(1, y)-p(1, y)) \mathrm{d} y-\int_{\Omega}|\nabla p|^{2} \mathrm{~d} x \mathrm{~d} y
$$

So, we have

$$
\begin{aligned}
&\left\langle A_{0}(f, p),(f, p)\right\rangle_{\left[L^{2}(\Omega)\right]^{2}}= \alpha_{p} \gamma_{p} \alpha_{f} \int_{\Omega}(\Delta f) f \mathrm{~d} x \mathrm{~d} y+\alpha_{f} \gamma_{f} \alpha_{p} \int_{\Omega}(\Delta p) p \mathrm{~d} x \mathrm{~d} y \\
&=-\alpha_{p} \gamma_{p} \alpha_{f} \gamma_{f} \int_{0}^{L} f(1, y)(f(1, y)-p(1, y)) \mathrm{d} y \\
&-\alpha_{p} \gamma_{p} \alpha_{f} \int_{\Omega}|\nabla f|^{2} \mathrm{~d} x \mathrm{~d} y \\
&+\alpha_{f} \gamma_{f} \alpha_{p} \gamma_{p} \int_{0}^{L} p(1, y)(f(1, y)-p(1, y)) \mathrm{d} y \\
&=-\alpha_{p} \gamma_{p} \alpha_{f} \gamma_{f} \int_{0}^{L}(f(1, y)-p(1, y))^{2} \mathrm{~d} y \\
&-\alpha_{f} \alpha_{p} \int_{\Omega}\left(\gamma_{p}|\nabla f|^{2}+\varphi_{f}|\nabla p|^{2}\right) \mathrm{d} x \mathrm{~d} y
\end{aligned}
$$


On the other hand

$$
\begin{aligned}
\left\langle B_{0}(f, p),(f, p)\right\rangle= & -\alpha_{p} \gamma_{p} \beta_{f} \int_{\Omega}\left(\partial_{y} f\right) f \mathrm{~d} x \mathrm{~d} y+\alpha_{f} \gamma_{f} \beta_{p} \int_{\Omega}\left(\partial_{y} p\right) p \mathrm{~d} x \mathrm{~d} y \\
=- & \frac{\alpha_{p} \gamma_{p} \beta_{f}}{2} \int_{0}^{1}\left(f^{2}(x, 1)-f^{2}(x, 0)\right) \mathrm{d} x \\
& \quad+\frac{\alpha_{g} \gamma_{f} \beta_{p}}{2} \int_{0}^{1}\left(p^{2}(x, 1)-p^{2}(x, 0)\right) \mathrm{d} x \\
= & -\frac{\alpha_{p} \gamma_{p} \beta_{f}}{2} \int_{0}^{1} f^{2}(x, 1) \mathrm{d} x-\frac{\alpha_{f} \gamma_{f} \beta_{p}}{2} \int_{0}^{1} p^{2}(x, 0) \mathrm{d} x .
\end{aligned}
$$

Inequalities (18) and (19) prove that $A_{0}+t B_{0}$ is dissipative for every $t \in[0,1]$; moreover, we have seen that $A_{0}$ is onto, this proves that $A_{0}$ is self-adjoint, as $A_{0}$ is dissipative, we can conclude that $A_{0}$ is m-dissipative.

Now, as in the proof of the previous theorem, we shall prove that there exists $\delta>0$ such that, if $A_{0}+t_{0} B_{0}$ (here $t_{0} \in[0,1]$ ) is m-dissipative, then $A_{0}+t B_{0}$ is also mdissipative for every $t \in[0,1]$ such that $\left|t-t_{0}\right| \leq \delta$. To this end, we need the following proposition.

Proposition 4. If the operator $A_{0}+t_{0} B_{0}\left(t_{0} \in[0,1]\right)$ is m-dissipative, then the operator $B_{0}\left(\mathrm{I}-\left(A_{0}+t_{0} B_{0}\right)\right)^{-1}$ is bounded, the bound being independent from $t_{0}$.

Proof. Notice that if $A_{0}+t_{0} B_{0}$ is m-dissipative, $\mathrm{I}-\left(A_{0}+t_{0} B_{0}\right)$ is invertible. We denote the inverse $\left(\mathrm{I}-\left(A_{0}+t_{0} B_{0}\right)\right)^{-1}$ by $R\left(t_{0}\right)$ and we take $(f, p)$ in $\left[L^{2}(\Omega)\right]^{2}$. We seek for an upper bound for $\left\|B_{0} R\left(t_{0}\right)(f, p)\right\|$. Let $(u, v)=R\left(t_{0}\right)(f, p)$, so that we have

$$
f=u-\alpha_{f} \Delta u+t_{0} \beta_{f} \frac{\partial u}{\partial y}, \quad \quad p=v-\alpha_{p} \Delta v-t_{0} \beta_{p} \frac{\partial v}{\partial y} .
$$

Now

$$
\begin{aligned}
\left\|B_{0} R\left(t_{0}\right)(f, p)\right\|^{2} & =\left\|B_{0}(u, v)\right\|^{2} \\
& =\alpha_{p} \gamma_{p} \int_{\Omega} \beta_{f}^{2}\left(\frac{\partial u}{\partial y}\right)^{2} \mathrm{~d} x \mathrm{~d} y+\alpha_{f} \gamma_{f} \int_{\Omega} \beta_{p}^{2}\left(\frac{\partial p}{\partial y}\right)^{2} \mathrm{~d} x \mathrm{~d} y \\
& \leq M\left(\gamma_{p} \int_{\Omega}\left(\frac{\partial u}{\partial y}\right)^{2} \mathrm{~d} x \mathrm{~d} y+\gamma_{f} \int_{\Omega}\left(\frac{\partial p}{\partial y}\right)^{2} \mathrm{~d} x \mathrm{~d} y\right)
\end{aligned}
$$

with $M=\max \left(\alpha_{p} \beta_{f}^{2}, \alpha_{f} \beta_{p}^{2}\right)$.

We shall rewrite these two integrals; first we have

$$
\begin{aligned}
\int_{\Omega}\left(\frac{\partial u}{\partial y}\right)^{2} \mathrm{~d} x \mathrm{~d} y & =\int_{0}^{1}\left[u(x, y) \frac{\partial u}{\partial y}(x, y)\right]_{y=0}^{y=L} \mathrm{~d} x-\int_{\Omega} u \frac{\partial^{2} u}{\partial y^{2}} \mathrm{~d} x \mathrm{~d} y \\
& =-\int_{\Omega} u \frac{\partial^{2} u}{\partial y^{2}} \mathrm{~d} x \mathrm{~d} y \\
& =-\frac{1}{\alpha_{f}} \int_{\Omega} u\left(u-\alpha_{f} \frac{\partial^{2} u}{\partial x^{2}}+t_{0} \beta_{f} \frac{\partial u}{\partial y}-f\right) \mathrm{d} x \mathrm{~d} y \quad \text { from (20). }
\end{aligned}
$$


Now, we have

$$
\begin{aligned}
\int_{\Omega} u \frac{\partial^{2} u}{\partial x^{2}} \mathrm{~d} x \mathrm{~d} y & =\int_{0}^{L}\left[u(x, y) \frac{\partial u}{\partial x}(x, y)\right]_{x=0}^{x=1} \mathrm{~d} y-\int_{\Omega}\left(\frac{\partial u}{\partial x}\right)^{2} \mathrm{~d} x \mathrm{~d} y \\
& =-\gamma_{f} \int_{0}^{L} u(1, y)(u(1, y)-v(1, y)) \mathrm{d} y-\int_{\Omega}\left(\frac{\partial u}{\partial x}\right)^{2} \mathrm{~d} x \mathrm{~d} y
\end{aligned}
$$

on the other hand

$$
\int_{\Omega} u \frac{\partial u}{\partial y} \mathrm{~d} x \mathrm{~d} y=\frac{1}{2} \int_{0}^{1} u^{2}(x, 1) \mathrm{d} x .
$$

Substituting equalities (23) and (24) into (22), we get

$$
\begin{aligned}
\int_{\Omega}\left(\frac{\partial u}{\partial y}\right)^{2} \mathrm{~d} x \mathrm{~d} y= & -\frac{1}{\alpha_{f}} \int_{\Omega} u^{2} \mathrm{~d} x \mathrm{~d} y-\gamma_{f} \int_{0}^{L} u(1, y)(u(1, y)-v(1, y)) \mathrm{d} y \\
& -\int_{\Omega}\left(\frac{\partial u}{\partial x}\right)^{2} \mathrm{~d} x \mathrm{~d} y-\frac{t_{0} \beta_{f}}{2 \alpha_{f}} \int u^{2}(x, L) \mathrm{d} x+\frac{1}{\alpha_{f}} \int_{\Omega} u f \mathrm{~d} x \mathrm{~d} y \\
\leq & -\frac{1}{\alpha_{f}} \int_{\Omega} u^{2} \mathrm{~d} x \mathrm{~d} y-\gamma_{f} \int_{0}^{L} u(1, y)(u(1, y)-v(1, y)) \mathrm{d} y \\
& +\frac{1}{\alpha_{f}} \int_{\Omega} u f \mathrm{~d} x \mathrm{~d} y \\
\leq & -\frac{1}{\alpha_{f}} \int_{\Omega} u^{2} \mathrm{~d} x \mathrm{~d} y-\gamma_{f} \int_{0}^{L} u(1, y)(u(1, y)-v(1, y)) \mathrm{d} y \\
& +\frac{1}{2 \alpha_{f}} \int_{\Omega}\left(u^{2}+f^{2}\right) \mathrm{d} x \mathrm{~d} y \\
\leq & -\gamma_{f} \int_{0}^{L} u(1, y)(u(1, y)-v(1, y)) \mathrm{d} y+\frac{1}{2 \alpha_{f}} \int_{\Omega} f^{2} \mathrm{~d} x \mathrm{~d} y .
\end{aligned}
$$

An analogous computation shows that

$$
\int_{\Omega}\left(\frac{\partial v}{\partial y}\right)^{2} \mathrm{~d} x \mathrm{~d} y \leq \gamma_{p} \int_{0}^{L} v(1, y)(u(1, y)-v(1, y)) \mathrm{d} y+\frac{1}{2 \alpha_{p}} \int_{\Omega} p^{2} \mathrm{~d} x \mathrm{~d} y .
$$

Substituting (25) and (26) into (21), we get

$$
\begin{array}{rl}
\| B_{0} & R\left(t_{0}\right)(f, p) \|^{2} \leq M \gamma_{p}\left(-\gamma_{f} \int_{0}^{L} u(1, y)(u(1, y)-v(1, y)) \mathrm{d} y+\frac{1}{2 \alpha_{f}} \int_{\Omega} f^{2} \mathrm{~d} x \mathrm{~d} y\right) \\
& +M \gamma_{f}\left(\gamma_{p} \int_{0}^{L} v(1, y)(u(1, y)-v(1, y)) \mathrm{d} y+\frac{1}{2 \alpha_{p}} \int_{\Omega} p^{2} \mathrm{~d} x \mathrm{~d} y\right) \\
& =-M \gamma_{f} \gamma_{p} \int_{0}^{L}(u(1, y)-v(1, y))^{2} \mathrm{~d} y+M\left(\frac{\gamma_{p}}{2 \alpha_{f}} \int_{\Omega} f^{2} \mathrm{~d} x \mathrm{~d} y+\frac{\gamma_{f}}{2 \alpha_{p}} \int_{\Omega} p^{2} \mathrm{~d} x \mathrm{~d} y\right) \\
& \leq M^{\prime}\|(f, p)\|_{\left[L^{2}(\Omega)\right]^{2}}^{2},
\end{array}
$$

where $M^{\prime}=\max \left(\frac{M}{2 \alpha_{f}^{2}}, \frac{M}{2 \alpha_{p}^{2}}\right)$.

We have proved that the operator $B_{0} R\left(t_{0}\right)$ is bounded, moreover its norm is less than or equal to $\sqrt{M^{\prime}}$, which is a bound independent from $t_{0}$. 
We are now ready to prove the main result of this section.

TheOrem 5. Operator $A$ is $m$-dissipative with respect to the inner product (13).

Proof. Assume that $A_{0}+t_{0} B_{0}$ is m-dissipative (with $t_{0} \in[0,1]$ ), a simple computation shows that we can write

$$
\begin{aligned}
\mathrm{I}-\left(A_{0}+t B_{0}\right) & =\mathrm{I}-\left(A_{0}+t_{0} B_{0}\right)+\left(t_{0}-t\right) B_{0} \\
& =\left(\mathrm{I}+\left(t-t_{0}\right) B_{0} R\left(t_{0}\right)\right)\left(\mathrm{I}-\left(A_{0}+t_{0} B_{0}\right)\right) .
\end{aligned}
$$

The bounded operator $\mathrm{I}+\left(t-t_{0}\right) B_{0} R\left(t_{0}\right)$ is invertible if $\left\|\left(t-t_{0}\right) B_{0} R\left(t_{0}\right)\right\|<1$, and this inequality is true if $\left|t-t_{0}\right|<1 / \sqrt{M^{\prime}}$. Thus, if $t$ satisfies this inequality, we can conclude from (27) that $\mathrm{I}-\left(A_{0}+t B_{0}\right)$ is invertible. Moreover as, from Proposition 3, we know that $A_{0}+t B_{0}$ is dissipative (for every $t \geq 0$ ), we can conclude that $A_{0}+t B_{0}$ it is m-dissipative for every $t \in[0,1]$ and such that $\left|t-t_{0}\right| \leq 1 / \sqrt{M^{\prime}}$. Observe now that $A_{0}+t_{0} B_{0}$ is m-dissipative with $t_{0}=0$ ( $c f$ Prop. 3 ), since any point of $[0,1]$ can be reached from 0 by a finite number of step of length $1 / \sqrt{M^{\prime}}$, we conclude that $A=A_{0}+B_{0}$ is m-dissipative.

4. Existence and uniqueness of the solution of system (11). In order to ensure the existence of a solution to system (11) appropriate regularity assumptions on the initial datum are required. We next sharpen the regularity of this data.

4.1. The operator $A$ with inhomogeneous boundary conditions. In this section, we consider the following systems of partial differential equations

$$
\left\{\begin{array}{lc}
\alpha_{f} \Delta f(x, y)-\beta_{f} \partial_{y} f(x, y)=0, & (x, y) \in \Omega \\
-\alpha_{p} \Delta p(x, y)+\beta_{p} \partial_{y} p(x, y)=0 & (x, y) \in \Omega \\
f(x, 0)=T_{f} & 0 \leq x \leq 1, \\
\partial_{x} f(0, y)=0 & 0 \leq y \leq L, \\
\partial_{y} f(, x, L)=0 & 0 \leq x \leq 1, \\
p(x, L)=T_{p} & 0 \leq x \leq 1, \\
\partial_{x} p(0, y)=0 & 0 \leq y \leq L, \\
\partial_{y} p(x, 0)=0 & 0 \leq x \leq 1, \\
\partial_{x} f(1, y)=-\gamma_{f}(f(1, y)-p(1, y)) & 0 \leq y \leq L, \\
\partial_{x} p(1, y)=\gamma_{p}(f(1, y)-p(1, y)) & 0 \leq y \leq L .
\end{array}\right.
$$

If $T_{f}=T_{p}=0$, this system writes $A(f, p)=0$ with $A$ the operator defined above; in this case the unique solution of the system is $(f, p) \equiv(0,0)$ because the m-dissipative operator $A: \mathcal{D}(A) \rightarrow\left[L^{2}(\Omega)\right]^{2}$ is invertible. In the general case, we shall use some tools from the theory of boundary control systems (see e.g. [25, Chap. 10]). In system (28), we shall regard $T_{f}$ and $T_{p}$ as boundary controls, and we introduce the following spaces and operators:

- the solution space $\mathbf{Z}$ is defined as those pairs $(f, p) \in H^{2}(\Omega) \times H^{2}(\Omega)$ satisfying the following homogeneous boundary conditions:

- for every $0 \leq y \leq L, \partial_{x} f(0, y)=\partial_{x} p(0, y)=0$;

- for every $0 \leq x \leq 1, \partial_{y} f(, x, L)=\partial_{y} p(x, 0)=0$;

- for every $0 \leq y \leq L, \partial_{x} f(1, y)=-\gamma_{f}(f(1, y)-p(1, y))$, and $\partial_{x} p(1, y)=$ $\gamma_{p}(f(1, y)-p(1, y))$.

- the state space $\mathbf{X}$ is the space $L^{2}(\Omega) \times L^{2}(\Omega)$; 
- the input space $\mathbf{U}$ is the space $L^{2}(0,1) \times L^{2}(0,1)$.

Notice that $\mathbf{Z} \subset \mathbf{X}$ with continuous embedding. We consider the operator $L: \mathbf{Z} \rightarrow \mathbf{X}$ defined as

$$
L(f, p)=\left(\alpha_{f} \Delta f-\beta_{f} \partial_{y} f, \alpha_{p} \Delta p+\beta_{p} \partial_{y} p\right)
$$

and the operator $G: \mathbf{Z} \rightarrow \mathbf{U}$ defined as

$$
G(f, p)=(f(\cdot, 0), p(\cdot, L)) .
$$

Operator $L$ is obviously bounded, this is true also for operator $G$

Lemma 6. The linear operator $G: \mathbf{Z} \rightarrow \mathbf{U}$ is bounded.

Proof. Consider the function $F$ defined for $x \in[0,1]$ as

$$
F(x)=\int_{0}^{L} f^{2}(x, y) \mathrm{d} y,
$$

we have

$$
\begin{aligned}
F(0) & =F(x)-\int_{0}^{x} \frac{\mathrm{d} F(\xi)}{\mathrm{d} \xi} \mathrm{d} \xi \\
& =F(x)-2 \int_{0}^{x}\left(\int_{0}^{L} f(\xi, y) \partial_{x} f(\xi, y) \mathrm{d} \xi\right) \mathrm{d} y \\
& \leq F(x)+2 \int_{0}^{1}\left(\int_{0}^{L}|f(\xi, y)|\left|\partial_{x} f(\xi, y)\right| \mathrm{d} \xi\right) \mathrm{d} y \\
& \leq F(x)+\int_{0}^{1}\left(\int_{0}^{L} f^{2}(\xi, y) \mathrm{d} \xi\right) \mathrm{d} y+\int_{0}^{1}\left(\int_{0}^{L}\left(\partial_{x} f\right)^{2}(\xi, y) \mathrm{d} \xi\right) \mathrm{d} y .
\end{aligned}
$$

By integrating this inequality with respect to $x$ on the interval $[0,1]$, we obtain

$$
F(0) \leq \int_{0}^{1} F(x) \mathrm{d} x+\int_{\Omega} f^{2} \mathrm{~d} x \mathrm{~d} y+\int_{\Omega}\left(\partial_{y} f\right)^{2} \mathrm{~d} x \mathrm{~d} y
$$

which reads

$$
\|f(\cdot, 0)\|_{L^{2}(0,1)} \leq 2\|f\|_{L^{2}(\Omega)}^{2}+\left\|\partial_{x} f\right\|_{L^{2}(\Omega)}^{2}
$$

and so we have

$$
\|f(\cdot, 0)\|_{L^{2}(0,1)} \leq 2\|f\|_{H^{2}(\Omega)} .
$$

Clearly, the same inequality is true for $p$, which proves that $G$ is a bounded operator. $\square$

Now we have.

Proposition 7. The operators Gand L satisfy the following properties.

1. $G$ is onto;

2. $\operatorname{Ker} G$ is dense in $\mathbf{X}$;

3. $-L$ restricted to $\operatorname{Ker} G$ is onto;

4. $\operatorname{Ker}(-L) \cap \operatorname{Ker} G=\{0\}$. 
The two first points are obvious. As regards the third point, notice first that $\operatorname{Ker} G \subset \mathcal{D}(A)$ moreover, given $(u, v) \in \mathbf{X}$, we know that there exist $(f, p) \in \mathcal{D}(A)$ such that $\mathcal{A}(f, p)=(u, v)$. In order to prove that the pair $(f, g)$ is in $\operatorname{Ker} \mathbf{G}$, we have to show that $f$ and $p$ are in $H^{2}(\Omega)$. The proof of the regularity of the weak solution of an elliptic equation is a classical result (see e.g. [8] or [2]) but this result assumes Dirichlet or Neumann boundary conditions. In [9], M. Faierman proves the regularity of the weak solution of an elliptic equation $M q=r$ where $M$ is an elliptic operator defined on a rectangle $R$ and where, as for the system considered in this paper, the boundary conditions are of mixed type: Dirichlet, Neumann of Robin. More specifically, under the condition that $r \in L^{2}(R)$, Faierman proves that $q$ is in $H^{2}(R)$. This proof is intended for an elliptic equation whose unknown function $f$ takes its values in $\mathbf{R}$ while, in this paper, our unknown is a couple of functions $(f, p)$; nevertheless, it suffices to adapt slightly the reasoning of Faierman to prove that $f$ and $p$ are in $H^{2}(\Omega)$.

Proposition 8. If both functions $u$ and $v$ are in $L^{2}(\Omega)$, the unique pair $(f, p) \in$ $\mathcal{D}(A)$ such that $A(f, p)=(u, v)$ belongs to $\mathcal{H}^{2}(\Omega) \times \mathcal{H}^{2}(\Omega)$.

Proof. Hereafter, we treat only the case of $f$, the reasoning for $p$ being the same than for $f$. First, we define two extensions of $f: f_{1}$ on $\Omega_{1}=[-1,1] \times[0, L]$ and $f_{2}$ on $\Omega_{2}=[0,2] \times[0, L]$ as follows

$$
\begin{aligned}
& f_{1}(x, y)= \begin{cases}\phi_{1}(x) f(x, y) & \text { if } 0 \leq x \leq 1 \\
\phi_{1}(-x) f(-x, y) & \text { if }-1 \leq x \leq 0\end{cases} \\
& f_{2}(x, y)= \begin{cases}\left(1-\phi_{1}(x)\right) f(x, y) & \text { if } 0 \leq x \leq 1 \\
-\left(1-\phi_{1}(2-x)\right) f(2-x, y)+2 f(1, y) & \text { if } 1 \leq x \leq 2\end{cases}
\end{aligned}
$$

where $\phi_{1}$ is a $C^{\infty}$ function such that

$$
\phi_{1}(x)= \begin{cases}1 & \text { if } x \leq 1 / 4 \\ 0 & \text { si } 3 / 4 \leq x \leq 1\end{cases}
$$

and $0 \leq \phi_{1}(x) \leq 1$ for all $x \in \mathbf{R}$. Notice that $f_{1}$ is defined as in [9] but the definition for $f_{2}$ (as well as the notations) differs slightly from the one adopted in this paper. First, it is easily shown that $f_{i} \in H^{2}\left(\Omega_{i}\right)(i=1,2)$, then we shall show that $f_{1}$ and $f_{2}$ can be regarded as weak solutions to some PDE's. We begin with function $f_{2}$ : take $\psi_{2} \in H^{1}\left(\Omega_{2}\right)$ such that $\psi_{2}(x, 0)=0$ for $0 \leq x \leq 2$, first notice that we have

$$
\begin{aligned}
\alpha_{f}\left(\nabla f_{2}\right)\left(\nabla \psi_{2}\right)+\beta_{f}\left(\partial_{y} f_{2}\right) \psi_{2}=\alpha_{f}(\nabla f)\left(\nabla\left(\phi_{2} \psi_{2}\right)\right)+\beta_{f}\left(\partial_{y} f\right)\left(\phi_{2} \psi_{2}\right) & \\
& -\alpha_{f}\left(\partial_{x} \phi_{2}\right)\left(\partial_{x} f\right) \psi_{2}+\alpha_{f}\left(\partial_{x} \phi_{2}\right) f\left(\partial_{x} \psi_{2}\right)
\end{aligned}
$$

where $\phi_{2}(x):=1-\phi_{1}(x)$. Integrating by parts and taking into account that $\mathcal{A}(f, p)=$ $(u, v)$, we obtain the following equality

$$
\begin{aligned}
\int_{\Omega} \alpha_{f}(\nabla f) \cdot\left(\nabla \psi_{2}\right) \mathrm{d} x \mathrm{~d} y & +\int_{\Omega} \beta_{f}\left(\partial_{y} f\right) \psi_{2} \mathrm{~d} x \mathrm{~d} y \\
& =-\int_{\Omega} u \psi_{2} \mathrm{~d} x \mathrm{~d} y+\alpha_{f} \int_{0}^{L} \partial_{x} f(1, y) \psi_{2}(1, y) \mathrm{d} y
\end{aligned}
$$


from this equality and (29), and taking into account that $\phi_{2}(1)=1$, and $\left(\partial_{x} \phi_{2}\right)(0)=$ $\left(\partial_{x} \phi_{2}\right)(1)=0$, we obtain

$$
\begin{aligned}
\int_{\Omega} \alpha_{f}\left(\nabla f_{2}\right) \cdot & \left(\nabla \psi_{2}\right) \mathrm{d} x \mathrm{~d} y+\int_{\Omega} \beta_{f}\left(\partial_{y} f_{2}\right) \psi_{2} \mathrm{~d} x \mathrm{~d} y \\
= & -\int_{\Omega}\left(\phi_{2} u+\alpha_{f}\left(\partial_{x} \phi_{2}\right)\left(\partial_{x} f\right)\right) \psi_{2} \mathrm{~d} x \mathrm{~d} y \\
& +\alpha_{f} \int_{0}^{L} \partial_{x} f(1, y) \psi_{2}(1, y) \mathrm{d} y+\int_{\Omega} \partial_{x} \phi_{2} f\left(\partial_{x} \psi_{2}\right) \mathrm{d} x \mathrm{~d} y \\
= & -\int_{\Omega}\left(\phi_{2} u+\alpha_{f}\left(\partial_{x} \phi_{2}\right)\left(\partial_{x} f\right)\right) \psi_{2} \mathrm{~d} x \mathrm{~d} y+\alpha_{f} \int_{0}^{L} \partial_{x} f(1, y) \psi_{2}(1, y) \mathrm{d} y \\
& +\alpha_{f} \int_{0}^{L}\left[\left(\partial_{x} \phi_{2}\right) f \psi_{2}\right]_{x=0}^{x=1} \mathrm{~d} y-\alpha_{f} \int_{\Omega}\left(\left(\partial_{x x} \phi_{2}\right) f+\left(\partial_{x} \phi_{2}\right)\left(\partial_{x} f\right)\right) \psi_{2} \mathrm{~d} x \mathrm{~d} y \\
= & \left.-\int_{\Omega}\left(\phi_{2} u+g_{2}\right) \psi_{2} \mathrm{~d} x \mathrm{~d} y+\alpha_{f} \int_{0}^{L}\left(\partial_{x} f\right)(1, y)\right) \psi_{2}(1, y) \mathrm{d} y
\end{aligned}
$$

where $g_{2}$ is the function defined as

$$
g_{2}(x, y):=\alpha_{f}\left(\partial_{x x} \phi_{2}(x) f(x, y)+2 \partial_{x} \phi_{2}(x) \partial_{x} f(x, y)\right) .
$$

From this formula, we deduce that,

$$
\begin{array}{r}
\int_{\Omega_{2}} \alpha_{f}\left(\nabla f_{2}\right) \cdot\left(\nabla \psi_{2}\right) \mathrm{d} x \mathrm{~d} y+\int_{\Omega_{2}} \beta_{f}\left(\partial_{y} f_{2}\right) \psi_{2} \mathrm{~d} x \mathrm{~d} y=-\int_{\Omega_{2}}\left(\phi_{2} u+g_{2}\right)^{*} \psi_{2} \mathrm{~d} x \mathrm{~d} y \\
+2 \alpha_{f} \int_{\Omega_{2}}\left(\partial_{y} f\right)(1, y)\left(\partial_{y} \psi_{2}\right)(x, y) \mathrm{d} x \mathrm{~d} y
\end{array}
$$

where

$$
\left(\phi_{2} u+g_{2}\right)^{*}:= \begin{cases}\left(\phi_{2} u+g_{2}\right)(x, y) & \text { if }(x, y) \in \Omega \\ -\left(\phi_{2} u+g_{2}\right)(2-x, y) & \text { if }(x, y) \in[1,2] \times[0, L] .\end{cases}
$$

Due to the second integral in the right-hand member in (31), function $f_{2}$ cannot be regarded as the weak solution of a PDE, nevertheless, we can apply the method of difference quotients. In the proof of [8, Th. 1, p. 329], an open set $V \subset \bar{V} \subset \Omega_{3}$ is fixed and a smooth cutoff function $\theta$ is chosen $(\theta$ is equal to 1 on $V$ and to 0 outside an open set $W$ such that $U \subset W \subset \bar{W} \subset \Omega_{3}$ ); then function $\psi_{2}$ in equality (31) is taken to be equal to

$$
\psi_{2}(x, y)=\frac{1}{h^{2}}\left(\theta ^ { 2 } ( x - h , y ) \left(f(x, y)-f(x-h, y)-\theta^{2}(x, y)(f(x+h, y)-f(x, y)) .\right.\right.
$$

With this choice of $\psi_{2}$, the second integral in the right-hand member of (31) is zero and it follows that we can argue as in the proof of [8, Th. 1, p. 329].

To prove the boundary regularity, we can still proceed as in [8], in this case also, we do not have to take care of the second integral in the right-hand member of (31). To be more precise, consider a point $\left(x_{0}, 0\right)$ of the edge $[0,2] \times\{0\}$ of $\Omega_{2}$ with $1 / 4 \leq x_{0} \leq 7 / 4$. Denote by $U_{r}$ the half ball $U_{r}=B\left(x_{0}, r\right) \cap \mathbf{R}_{+}^{2}$ where, as usual, $B\left(x_{0}, r\right)$ denotes the open ball of radius $r$ centered at $x_{0}$ and $\mathbf{R}_{+}^{2}=\left\{(x, y) \in \mathbf{R}^{2} \mid y>0\right\}$; moreover $r$ is 
chosen small enough in order that $B\left(x_{0}, 2 r\right)$ does not intersect the edge $[0,2] \times\{L\}$. Select a smooth cutoff function $\sigma$ satisfying

$$
\left\{\begin{array}{l}
\sigma \equiv 1 \text { on } B\left(x_{0}, r\right), \quad \sigma \equiv 0 \text { on } \mathbf{R}^{2} \backslash B\left(x_{0}, 2 r\right) \\
0 \leq \sigma \leq 1 .
\end{array}\right.
$$

Let $h>0$ be small and write

$$
\psi_{2}(x, y):=-D_{1}^{-h}\left(\sigma^{2} D_{1}^{h} K^{a}\right)
$$

where, for any function $K, D_{1}^{h} K$ denotes the difference quotient

$$
D_{1}^{h} K(x, y):=\frac{K(x+h)-K(x)}{h} .
$$

With this choice of $\psi_{2}$, it is easily seen that the second integral in the right-hand member of (31) vanishes. Thus we can argue exactly as in the proof of [8, Th.4, p. $336]$ in order to establish the following estimate

$$
\int_{U}\left\|D_{1}^{h} \nabla f_{2}\right\|^{2} \mathrm{~d} x \mathrm{~d} y \leq C
$$

which proves the result. We treat the regularity near the piece of boundary $[1,2] \times\{L\}$ in the same way and we notice that we do not have to worry about the corner since $f_{2}$ is zero in some neighborhoods of the edges $\{1\} \times[0, L]$ and $\{2\} \times[0, L]$.

The case of $f_{1}$ is slightly simpler, take $\psi_{1}$ in $H^{1}\left(\Omega_{1}\right)$ and such that $\psi_{1}(x, 0)=0$ for $-1 \leq x \leq 1$, we have where $g_{3}$ is defined similarly as $g_{1}$. We obtain for $f_{1}$ a formula analogous to (29), from this formula and (30), we get

$$
\int_{\Omega} \alpha_{f}\left(\nabla f_{1}\right) \cdot\left(\nabla \psi_{1}\right) \mathrm{d} x \mathrm{~d} y+\int_{\Omega} \beta_{f}\left(\partial_{y} f_{1}\right) \psi_{1} \mathrm{~d} x \mathrm{~d} y=-\int_{\Omega}\left(\phi_{1} u+g_{1}\right) \psi_{1} \mathrm{~d} x \mathrm{~d} y ;
$$

notice that in this case, as $\phi_{1}(1)=0$, we do not have to deal with a term like the second integral in the right-hand member of (31). From this equality, we deduce

$$
\int_{\Omega_{1}} \alpha_{f}\left(\nabla f_{1}\right) \cdot\left(\nabla \psi_{1}\right) \mathrm{d} x \mathrm{~d} y+\int_{\Omega_{1}} \beta_{f}\left(\partial_{y} f_{1}\right) \psi_{1} \mathrm{~d} x \mathrm{~d} y=-\int_{\Omega_{1}}\left(\phi_{1} u+g_{1}\right)^{*} \psi_{1} \mathrm{~d} x \mathrm{~d} y
$$

here $g_{1}$ and $\left(\phi_{1} u+g 1\right)^{*}$ are defined analogously as $g_{2}$ and $\left(\phi_{2} u+g_{2}\right)^{*}$. These computations show that $f_{1}$ is a weak solution of the following problem:

$$
\begin{gathered}
\alpha_{f} \Delta f_{1}-\beta_{f} \partial_{y} f_{1}=\left(u \phi_{1}+g_{1}\right)^{*} \text { on } \Omega_{1} \\
f_{1}=0 \text { on } \partial \Omega_{1} \backslash \Gamma_{1} \\
\frac{\mathrm{d} f_{1}}{\mathrm{~d} \nu}=0 \text { on } \Gamma_{1}
\end{gathered}
$$

here, as usual, $\mathrm{d} f_{i} / \mathrm{d} \nu$ denotes the normal derivative and $\Gamma_{1}$ is the edge of the rectangle $\Omega_{1}$ defined as $\Gamma_{1}=[-1,1] \times\{L\}$. Classical results (see e.g. [8]) allow us to assert that $f_{1}$ is in $H_{\text {loc }}^{2}\left(\Omega_{i}\right)$. Concerning the regularity up to the boundary, we can argue exactly as in in [8, Th. 4, p. 336]; thus, function $f_{1}$ is in $H^{2}\left(\Omega_{1}\right)$. Consider now the function $f_{1}+f_{2}$ restricted to $\Omega$, this function is in $H^{2}(\Omega)$ and is equal to $f$, thus we proved that $f \in H^{2}(\Omega)$. 
Take now $(f, p)$ in $\operatorname{Ker}(-L) \cap \operatorname{Ker} G,(f, p)$ belongs to $\mathcal{D}(A)$, so $L(f, p)=(0,0)$ implies $A(f, p)=(0,0)$ which in turn implies $(f, p)=(0,0)$ because $A$ is injective. This achieve the proof of Theorem 7 .

Thus, we can apply [25, Proposition 10.1.2]: there exist a unique operator $B \in$ $\mathcal{L}\left(\mathbf{U}, \mathbf{X}_{-1}\right)$ such that

$$
L=\mathcal{A}+B G .
$$

Here $\mathcal{A}$ denotes the restriction of $L$ to $\operatorname{Ker} G$ and is thus identical to the operator $A$ defined in section $3 ; \mathbf{X}_{-1}$ denotes the completion of the space $\mathbf{X}$ with respect to the norm $\|(u, v)\|_{-1}=\left\|\mathcal{A}^{-1}(u, v)\right\|$.

Operator $A$ is m-dissipative, therefore, it is the generator of a contraction semigroup. Moreover from equalities (18) and (19), we obtain

$$
\langle A(f, p),(f, p)\rangle_{\left[L^{2}(\Omega)\right]^{2}} \leq-\left(\|\nabla f\|^{2}+\|\nabla p\|^{2}\right)
$$

from the Poincaré's inequality, we have

$$
\int_{\Omega}\left(\|\nabla\|^{2}+\|\nabla p\|^{2}\right) \mathrm{d} x \mathrm{~d} y \geq C \int_{\Omega}\left(f^{2}+p^{2}\right) \mathrm{d} x \mathrm{~d} y,
$$

which implies that $A \leq-C$ I. Denoting by $\mathbb{T}_{t}$ the semigroup generated by $A$, we thus have $\left\|\mathbb{T}_{t}\right\| \leq e^{-C t}$. As $A$ is the generator of a strongly continuous semigroup, for every $T>0,\left(f_{0}, p_{0}\right) \in \mathbf{Z}$, and $(u, v) \in \mathbf{U}$ such that $G\left(f_{0}, p_{0}\right)=(u, v)$, the equation

$$
\begin{aligned}
\frac{\mathrm{d}(f, p)}{\mathrm{d} t} & =L(f, p)=A(f, p+B G(u, v), \\
(f(0), p(0)) & =\left(f_{0}, p_{0}\right)
\end{aligned}
$$

admits a unique solution $(f, p)$ such that $(f, p) \in C([0, T] ; \mathbf{Z}) \cap C^{1}([0, T] ; \mathbf{X})$. Moreover as $\mathbb{T}_{t}$ is exponentially stable, we have $\lim _{t \rightarrow \infty}(f(t), p(t))=\left(f_{\infty}, p_{\infty}\right)$ where $\left(f_{\infty}, p_{\infty}\right)$ is the unique solution of the equation $B G\left(f_{0}, p_{0}\right)=-A\left(f_{\infty}, p_{\infty}\right)$. Thus, we have proved that, given any pair of initial conditions $\left(f_{0}, p_{0}\right)$ and any pair of input temperatures $\left(T_{f}, T_{p}\right)$, there exists a unique solution to system (11) which tends exponentially towards an asymptotic state $\left(f_{\infty}, p_{\infty}\right)$ as $t$ tends to infinity.

5. Co-current operator. In this section, we consider a DCMD model with cocurrent; the equations modeling this device are the same as (11) except for the sign of $\beta_{p}$ : they write

$$
\left\{\begin{array}{lr}
\partial_{t} f(t, x, y)-\alpha_{f} \Delta f(t, x, y)+\beta_{f} \partial_{y} f(t, x, y)=0, & t \geq 0,(x, y) \in \Omega \\
\partial_{t} p(t, x, y)-\alpha_{p} \Delta p(t, x, y)+\beta_{p} \partial_{y} p(t, x, y)=0 & t \geq 0,(x, y) \in \Omega \\
f(t, x, 0)=T_{f} & t \geq 0,0 \leq x \leq 1, \\
\partial_{x} f(t, 0, y)=0 & t \geq 0,0 \leq y \leq L, \\
\partial_{y} f(t, x, L)=0 & t \geq 0,0 \leq x \leq 1, \\
p(t, x, 0)=T_{p} & t \geq 0,0 \leq x \leq 1, \\
\partial_{x} p(t, 0, y)=0 & t \geq 0,0 \leq y \leq L, \\
\partial_{y} p(t, x, L)=0 & t \geq 0,0 \leq x \leq 1, \\
\partial_{x} f(t, 1, y)=-\gamma_{f}(f(t, 1, y)-p(t, 1, y)) & t \geq 0,0 \leq y \leq L, \\
\partial_{x} p(t, 1, y)=\gamma_{p}(f(t, 1, y)-p(t, 1, y)) & t \geq 0,0 \leq y \leq L, \\
f(0, x, y)=f_{0}(x, y) & (x, y) \in \Omega, \\
p(0, x, y)=p_{0}(x, y) & (x, y) \in \Omega .
\end{array}\right.
$$


Introducing the following change of variables

$$
g(t, x, y)=f(t, x, y) e^{-\frac{\beta_{f}}{2 \alpha_{f}} y}, \quad q(t, x, y)=p(t, x, y) e^{-\frac{\beta_{p}}{2 \alpha_{p}} y} .
$$

we then have

$$
\begin{aligned}
\partial_{x x} g(t, x, y) & =\partial_{x x} f(t, x, y) \exp \left(-\frac{\beta_{f}}{2 \alpha_{f}} y\right) \\
\partial_{y} g(t, x, y) & =\left(\partial_{y} f(t, x, y)-\frac{\beta_{f}}{2 \alpha_{f}} f(x, y)\right) \exp \left(-\frac{\beta_{f}}{2 \alpha_{f}} y\right), \\
\partial_{y y} g(t, x, y) & =\left(\partial_{y y} f(t, x, y)-\frac{\beta_{f}}{\alpha_{f}} \partial_{y} f(t, x, y)\right) \exp \left(-\frac{\beta_{f}}{2 \alpha_{f}} y\right)+\frac{\beta_{f}^{2}}{4 \alpha_{f}^{2}} g(t, x, y),
\end{aligned}
$$

and so

$$
\left(\alpha_{f} \Delta f(t, x, y)-\beta_{f} \partial_{y} f(t, x, y)\right) \exp \left(-\frac{\beta_{f}}{2 \alpha_{f}} y\right)=\alpha_{f} \Delta g(t, x, y)-\frac{\beta_{f}^{2}}{4 \alpha_{f}} g(t, x, y)
$$

Similar computations lead to

$$
\left(\alpha_{p} \Delta p(t, x, y)-\beta_{p} \partial_{y} p(t, x, y)\right) \exp \left(-\frac{\beta_{p}}{2 \alpha_{p}} y\right)=\alpha_{p} \Delta q(t, x, y)-\frac{\beta_{p}^{2}}{4 \alpha_{p}} q(t, x, y) .
$$

Regarding the boundary conditions, we have

$$
\begin{aligned}
g(t, x, 0) & =T_{f} \\
\partial_{x} g(t, 0, y) & =0 \\
\partial_{y} g(t, x, L) & =-\frac{\beta_{f}}{2 \alpha_{f}} g(t, x, L) \\
q(t, x, 0) & =T_{p} \\
\partial_{x} q(t, 0, y) & =0 \\
\partial_{y} q(t, x, L) & =-\frac{\beta_{p}}{2 \alpha_{p}} q(t, x, L) \\
\partial_{x} g(t, 1, y) & =-\gamma_{f}\left(g(t, 1, y)-q(t, 1, y) e^{\left(-\frac{\beta_{f}}{2 \alpha_{f}}+\frac{\beta_{p}}{2 \alpha_{p}}\right) y}\right) \\
\partial_{x} q(t, 1, y) & =\partial_{x} p(t, 1, y) e^{-\frac{\beta_{p}}{2 \alpha_{p}} y}=\gamma_{p}\left(e^{\left(\frac{\beta_{f}}{2 \alpha_{f}}-\frac{\beta_{p}}{2 \alpha_{p}}\right) y} g(1, y)-q(1, y)\right) .
\end{aligned}
$$

We assume that the flow velocities can be adjusted in such a way that

$$
\frac{\beta_{f}}{2 \alpha_{f}}=\frac{\beta_{p}}{2 \alpha_{p}}
$$

Under the change of unknown functions (34) and with the assumption (35), sys- 
tem (33) becomes

$$
\left\{\begin{array}{lr}
\partial_{t} g(t, x, y)-\alpha_{f} \Delta g(t, x, y)+\frac{\beta_{f}^{2}}{4 \alpha_{f}} g(t, x, y)=0, & t \geq 0,(x, y) \in \Omega \\
\partial_{t} q(t, x, y)-\alpha_{p} \Delta q(t, x, y)+\frac{\beta_{p}^{2}}{4 \alpha_{p}} q(t, x, y)=0 & t \geq 0,(x, y) \in \Omega \\
g(t, x, 0)=T_{f} & t \geq 0,0 \leq x \leq 1, \\
\partial_{x} g(t, 0, y)=0 & t \geq 0,0 \leq y \leq L, \\
\partial_{y} g(t, x, L)=-\frac{\beta_{f}}{2 \alpha_{f}} g(t, x, L) & t \geq 0,0 \leq x \leq 1, \\
q(t, x, 0)=T_{p} & t \geq 0,0 \leq x \leq 1, \\
\partial_{x} q(t, 0, y)=0 & t \geq 0,0 \leq y \leq L, \\
\partial_{y} q(t, x, 0)=-\frac{\beta_{p}}{2 \alpha_{p}} q(t, x, 0) & t \geq 0,0 \leq x \leq 1, \\
\partial_{x} g(t, 1, y)=-\gamma_{f}(g(t, 1, y)-q(t, 1, y)) & t \geq 0,0 \leq y \leq L, \\
\partial_{x} q(t, 1, y)=\gamma_{p}(g(t, 1, y)-q(t, 1, y)) & t \geq 0,0 \leq y \leq L, \\
g(0, x, y)=g_{0}(x, y):=f_{0}(x, y) e^{\left(-\frac{\beta_{f}}{2 \alpha_{f}} y\right)} & (x, y) \in \Omega, \\
q(0, x, y)=q_{0}(x, y):=p_{0}(x, y) e^{\left(-\frac{\beta_{p}}{2 \alpha_{p}} y\right)} & (x, y) \in \Omega .
\end{array}\right.
$$

We shall prove no that the operator, denoted by $\tilde{A}$, and related to this system is diagonalizable. The spaces related to this operator will be slightly different from the ones related to operator $A$. First, we introduce the space $\tilde{\mathbf{E}}$ defined as the set of those pairs $(g, q)$ in $\left[H^{1}(\Omega) \cap C^{1}(\bar{\Omega})\right]^{2}$ such that

- $g(x, 0)=q(x, 0)=0$ for every $x \in(0,1)$;

- $\partial_{x} g(0, y)=\partial_{x} q(0, y)=0$, for every $y \in(0, L)$;

- $\partial_{y} g(x, L)=-\frac{\beta_{f}}{2 \alpha_{f}} g(x, L)$ for every $x \in(0,1)$;

- $\partial_{y} q(x, L)=-\frac{\beta_{p}}{2 \alpha_{p}} q(x, L)$ for every $x \in(0,1)$;

- $\partial_{x} g(1, y)=-\gamma_{f}(g(1, y)-q(1, y))$, for every $y \in(0, L)$;

- $\partial_{x} q(1, y)=\gamma_{p}(g(1, y)-q(1, y))$ for every $y \in(0, L)$.

As section 3 , the space $H^{1}(\Omega) \times H^{1}(\Omega)$ is equipped with the product topology, making it an Hilbert space whose norm is defined by (12). On $L^{2}(\Omega) \times L^{2}(\Omega)$, we consider again the inner product given by (13). We denote then by $\tilde{\mathcal{H}}_{\mathrm{bc}}^{1}$ the closure of $\tilde{\mathbf{E}}$ in $\left[H^{1}(\Omega)\right]^{2}$; recall that the induced norm on $\tilde{\mathcal{H}}_{\mathrm{bc}}^{1}$ is also defined by (14).

The operator $\tilde{A}$ is then defined as follows: its domain is given by

$$
\mathcal{D}(\tilde{A}):=\left\{(g, q) \in \tilde{\mathcal{H}}_{\mathrm{bc}}^{1} \mid(\Delta g, \Delta q) \in\left[L^{2}(\Omega)\right]^{2}\right\} ;
$$

and, for every $(g, q) \in \mathcal{D}(\tilde{A})$,

$$
\tilde{A}(g, q)=\left(\alpha_{f} \Delta g-\frac{\beta_{f}^{2}}{4 \alpha_{f}} g, \alpha_{p} \Delta q-\frac{\beta_{p}^{2}}{4 \alpha_{p}}\right) .
$$

Using similar arguments as in section 3.1 , we can prove that $\tilde{A}$ is m-dissipative and diagonalizable; moreover reasoning as in section 4 , we can prove that, given an initial condition in $\mathcal{D}(\tilde{A})$, system (36) has a unique solution and that this solution converges, as $t \rightarrow \infty$, towards the solution $\left(g_{\infty}, q_{\infty}\right)$ of the equation $\tilde{B} \tilde{G}\left(g_{0}, q_{0}\right)=-\tilde{A}\left(g_{\infty}, q_{\infty}\right)$. 
6. Output Tracking for 2D Direct Contact Membrane Distillation. In this section, we are interested in the problem of performance output tracking for the DCMD system. The aim of this part is to propose an output feedback law in presence of unknown disturbance. We use the active disturbance rejection control (ADRC) method. We start our analysis by the co-current configuration, The counter-current configuration will be briefly discussed in the conclusion. The system (33) can be written in the matrix form

$$
\begin{cases}\partial_{t} w(t, x)-\alpha \Delta w(t, x)+B . \nabla w(t, x)=0 & t>0 x \in \Omega, \\ \frac{\partial w(t, x)}{\partial \nu}=\mathcal{M} w(t, x) & t>0 x \in \Gamma_{4}, \\ \frac{\partial w(t, x)}{\partial \nu}=u(t) & t>0 x \in \Gamma_{3}, \\ \frac{\partial w(t, x)}{\partial \nu}=0 & t>0 x \in \Gamma_{2}, \\ \frac{\partial w(t, x)}{\partial \nu}=d(t) & t>0 x \in \Gamma_{1}, \\ w(0, x)=w_{0}(x) & x \in \Omega, \\ w(t, x)=y_{m}(t, x) & t>0 x \in \Gamma_{1}, \\ y_{r}(t, x)=w(t, x) & t>0 x \in \Gamma_{3},\end{cases}
$$

where $\Gamma_{1}=(0,1) \times\{0\}, \Gamma_{2}=\{0\} \times(0, L), \Gamma_{3}=(0,1) \times\{L\}$ and $\Gamma_{4}=\{1\} \times(0, L)$, $w(t, x)=[f(t, x), p(t, x)]^{T}$, with the input $u(t)=\left[u_{1}(t, x), u_{2}(t, x)\right]^{T}$, as well as the unknown disturbance $d(t) . B=\left[\begin{array}{cc}0 & \beta_{f} \\ 0 & \beta_{p}\end{array}\right], \alpha=\left[\begin{array}{cc}\alpha_{f} & 0 \\ 0 & \alpha_{p}\end{array}\right], \mathcal{M}=\left[\begin{array}{cc}\gamma_{f} & -\gamma_{f} \\ -\gamma_{p} & \gamma_{p}\end{array}\right]$, and $y_{m}$ is the measured output, $y_{r}$ the performance output signal to be regulated. The system (37) is discussed on the boundary space $\mathcal{H}_{\Gamma_{i}}=\left[L^{2}\left(\Gamma_{i}\right)\right]^{2}$, for all $i \in\{1,2,3,4\}$. We denote by $\mathcal{H}_{s}^{m}=\left[H^{m}(\Omega)\right]^{2}$ the Sobolev space of order $m$ and $\mathcal{H}_{s \Gamma_{i}}^{m}=\left[H^{m}\left(\Gamma_{i}\right)\right]^{2}$ the Sobolev space of order $m$ on the boundary for all $i \in\{1,2,3,4\}$. Now, we give some boundary estimation on the error system. Our goal is to design an output feedback control for uncertain system (37) to reject the external disturbance and to achieve output tracking:

$$
\|e(t)\|_{\mathcal{H}_{\Gamma_{3}}}=\left\|y_{r}(t)-r(t)\right\|_{\mathcal{H}_{\Gamma_{3}}} \longrightarrow 0 \quad \text { as } t \rightarrow \infty,
$$

where $r$ is a reference signal. The idea in this section consists to propose a feedback law based on the heat flux at the output of the feed and the permeate in order to obtain the desire output temperature. This desired temperature design the well running temperature of the DCMD system. The input temperature of the system $T_{f}$ and $T_{p}$ in (33) are the measurements of the system. However, the system is suffered to an unknown input heat flux disturbance $d(t)$ in the input of the system.

6.1. State observer design. We now design an extended state observer (ESO) that can estimate not only the the state $w(t, x)$ of the controlled system (37) but also 
the disturbance $d(t)$. The ESO is designed as follows;

$$
\begin{cases}\partial_{t} \hat{w}(t, x)-\alpha \Delta \hat{w}(t, x)+B . \nabla \hat{w}(t, x)=0 & t>0 x \in \Omega, \\ \frac{\partial \hat{w}(t, x)}{\partial \nu}=\mathcal{M} \hat{w}(t, x) & t>0 x \in \Gamma_{4}, \\ \frac{\partial \hat{w}(t, x)}{\partial \nu}=u(t, x) & t>0 x \in \Gamma_{3}, \\ \frac{\partial \hat{w}(t, x)}{\partial \nu}=0 & t>0 x \in \Gamma_{2}, \\ \hat{w}(t, x)=y_{m}(t, x) & t>0 x \in \Gamma_{1}, \\ \hat{w}(0, x)=\hat{w}_{0}(x) & x \in \Omega .\end{cases}
$$

The error $\tilde{w}(t, x)=\hat{w}(t, x)-w(t, x)$ is governed by

$$
\begin{cases}\partial_{t} \tilde{w}(t, x)-\alpha \Delta \tilde{w}(t, x)+B . \nabla \tilde{w}(t, x)=0 & t>0 x \in \Omega, \\ \frac{\partial \tilde{w}(t, x)}{\partial \nu}=\mathcal{M} \tilde{w}(t, x) & t>0 x \in \Gamma_{4}, \\ \frac{\partial \tilde{w}(t, x)}{\partial \nu}(t, x)=0 & t>0 x \in \Gamma_{3}, \\ \frac{\partial \tilde{w}(t, x)}{\partial \nu}=0 & t>0 x \in \Gamma_{2}, \\ \tilde{w}(t, x)=0 & t>0 x \in \Gamma_{1}, \\ \tilde{w}(0, x)=\tilde{w}_{0}(x) & x \in \Omega .\end{cases}
$$

Now, we give some boundary estimation on the error system.

Lemma 9. Let us assume $\tilde{w}_{0} \in \mathbf{X}$, the system (39) admits a unique solution $\tilde{w} \in C(0, \infty ; \mathbf{X})$ and there exists $\delta_{0}>0$ such that

$$
\|\tilde{w}(t)\|_{\mathbf{x}} \leqslant e^{-\delta_{0} t}\left\|\tilde{w}_{0}\right\|_{\mathbf{x}}, \quad \forall t>0
$$

Moreover, for any $\tau>0$ there exists $M_{0}>0$ depending on $\tau$ and $\delta_{0}>0$ such that

$$
\left\|\frac{\partial \tilde{w}}{\partial \nu}\right\|_{\mathcal{H}_{\Gamma_{1}}} \leqslant M_{0} e^{-\delta_{0} t}\left\|\tilde{w}_{0}\right\|_{\mathbf{x}}, \quad\|\tilde{w}\|_{\mathcal{H}_{\Gamma_{3}}} \leqslant M_{0} e^{-\delta_{0} t}\left\|\tilde{w}_{0}\right\|_{\mathbf{X}}, \quad \forall t>\tau .
$$

Proof. Let introduce the operator

$$
\tilde{\mathcal{A}}=\Delta \phi+B . \nabla \phi, \forall \phi \in D(\tilde{\mathcal{A}}),
$$

and

$$
D(\tilde{\mathcal{A}})=\left\{\phi \in \mathbf{Z} ;\left.\frac{\partial \phi}{\partial \nu}\right|_{\Gamma_{4}}=\mathcal{M} \phi ;\left.\frac{\partial \phi}{\partial \nu}\right|_{\Gamma_{2}}=\left.\frac{\partial \phi}{\partial \nu}\right|_{\Gamma_{3}}=\left.\phi\right|_{\Gamma_{1}}=0\right\}
$$

From section 4 , by [23, Theorem 2.7 p.211] it is easy to show that $\tilde{\mathcal{A}}$ generates an analytical semigroup $\tilde{S}(t)$ which implies that (39) admits a unique solution

$$
\tilde{w}=\tilde{S} \tilde{w}_{0} \in C(0, \infty ; \mathbf{X}) .
$$

Now, we show that the semi-group $\tilde{S}$ is exponentially stable. Indeed, we introduce the following Lyapunov function

$$
V_{0}(t)=\frac{1}{2} \alpha_{p} \gamma_{p} \int_{\Omega} \tilde{w}_{1}(t, x) d x+\frac{1}{2} \alpha_{f} \gamma_{f} \int_{\Omega} \tilde{w}_{2}(t, x) d x .
$$


Differentiating $V_{0}$ along the solution of (39) and using Green's formula and Poincarée inequality we deduce that there exists $\delta>0$ such that

$$
\dot{V}_{0}(t) \leqslant-\delta V_{0}(t)
$$

which give the exponential stability of $\tilde{S}$,i.e.

$$
\|\tilde{w}(t)\|_{\mathbf{x}}=\left\|\tilde{S}(t) \tilde{w}_{0}\right\|_{\mathbf{x}} \leqslant e^{-\delta t}\left\|\tilde{w}_{0}\right\|_{\mathbf{x}}
$$

Therefore, $\tilde{S}(t)$ is an analytic semigroup, see [23], from any positive integer $m$, we have $\tilde{S}(t) \tilde{w}_{0} \in D(\tilde{\mathcal{A}})$ for all $t>0$ and there exists a constant $C>0$ such that

$$
\|\tilde{\mathcal{A}} \tilde{S}(t)\| \leqslant \frac{C}{t}, \forall t>0
$$

So, we obtain $\tilde{\mathcal{A}}^{m} \tilde{S}(t) \tilde{w}_{0}=(\tilde{\mathcal{A}} S(t / m))^{m} \tilde{w}_{0}$, by (42) it follows that

$$
\begin{aligned}
\left\|\Delta^{m} \tilde{w}(t, .)\right\|_{\mathbf{X}} & =\left\|\tilde{\mathcal{A}}^{m} \tilde{w}(t, .)\right\|_{\mathbf{X}}=\left\|(\tilde{\mathcal{A}} S(t / m))^{m} \tilde{w}_{0}\right\|_{\mathbf{X}} \\
& \leqslant \frac{C^{m} m^{m}}{t^{m}}\left\|\tilde{w}_{0}\right\|_{\mathbf{X}} .
\end{aligned}
$$

Moreover, by using the Sobolev embedding theorem, there exists $C_{1}>0$ such that

$$
\begin{aligned}
\|\tilde{w}(t)\|_{\mathcal{H}_{s}^{2 m}} & \leqslant C_{1}\left(\left\|\Delta^{m} \tilde{w}(t)\right\|_{\mathbf{X}}+\|\tilde{w}(t)\|_{\mathbf{X}}\right) \\
& \leqslant\left(\frac{C_{1} C^{m} m^{m}}{t^{m}}+C_{1} M_{0} e^{-\mu t}\right)\left\|\tilde{w}_{0}\right\|_{\mathbf{X}} .
\end{aligned}
$$

The Sobolev trace theorem implies that

$$
\left\|\frac{\partial \tilde{w}(t)}{\partial \nu}\right\|_{\mathcal{H}_{\Gamma_{3}}} \leqslant C_{3}\|\tilde{w}\|_{\mathcal{H}_{s}^{2 m}}, \quad\left\|\frac{\partial \tilde{w}(t)}{\partial \nu}\right\|_{\mathcal{H}_{\Gamma_{1}}} \leqslant C_{3}\|\tilde{w}\|_{\mathcal{H}_{s}^{2 m}}
$$

for some constant $C_{3}>0$. Therefore, by (40) we get

$$
\begin{aligned}
\left\|\Delta^{m} \tilde{w}(t)\right\|_{\mathbf{X}} & =\left\|\tilde{\mathcal{A}}^{m} \tilde{w}(t)\right\|_{\mathbf{X}}=\left\|\tilde{\mathcal{A}}^{m} \tilde{S}(t) \tilde{w}_{0}\right\|_{\mathbf{X}} \\
& =\left\|\tilde{S}(t-\tau) \tilde{\mathcal{A}} \tilde{S}(\tau) \tilde{w}_{0}\right\|_{\mathbf{X}} \\
& \leqslant M e^{-\mu(t-\tau)}\left\|\tilde{\mathcal{A}} \tilde{S}(t) \tilde{w}_{0}\right\|_{\mathbf{X}}
\end{aligned}
$$

for any $\tau>0$. Finally, from (43) and the Sobolev embedding theorem, it follows the estimates in (40).

6.2. Servomechanism. We design a servomechanism for system (37) in term of the reference signal $r(t, x)$.

$$
\begin{cases}\partial_{t} v(t, x)-\alpha \Delta v(t, x)+B . \nabla v(t, x)=0 & t>0 x \in \Omega, \\ \frac{\partial v(t, x)}{\partial \nu}=\mathcal{M} v(t, x) & t>0 x \in \Gamma_{4}, \\ v(t, x)=r(t) & t>0 x \in \Gamma_{3}, \\ \frac{\partial v(t, x)}{\partial \nu}=0 & t>0 x \in \Gamma_{2}, \\ v(t, x)=\hat{w}(t, x) & t>0 x \in \Gamma_{1}, \\ v(0, x)=v_{0}(x) & x \in \Omega .\end{cases}
$$


The error tracking is given by

(45)

$$
\begin{aligned}
e(t, x)=y_{r}(t, x)-r(t, x) & =w(t, x)_{\left.\right|_{\Gamma_{3}}}-v(t, x)_{\left.\right|_{\Gamma_{3}}} \\
& =\left(w(t, x)_{\left.\right|_{\Gamma_{3}}}-\hat{w}(t, x)_{\left.\right|_{\Gamma_{3}}}\right)+\left(\hat{w}(t, x)_{\left.\right|_{\Gamma_{3}}}-v(t, x)_{\left.\right|_{\Gamma_{3}}}\right) .
\end{aligned}
$$

Let us consider now the error equation $\eta(t, x)=v(t, x)-\hat{w}(t, x)$ between the state of servo-system and the state observer

$$
\begin{cases}\partial_{t} \eta(t, x)-\alpha \Delta \eta(t, x)+B . \nabla \eta(t, x)=0 & t>0 \quad x \in \Omega, \\ \frac{\partial \eta(t, x)}{\partial \nu}=\mathcal{M} \eta(t, x) & t>0 x \in \Gamma_{4}, \\ \frac{\partial \eta(t, x)}{\partial \nu}=\frac{\partial v(t, x)}{\partial \nu}-u(t, x) & t>0 x \in \Gamma_{3}, \\ \frac{\partial \eta(t, x)}{\partial \nu}=0 & t>0 x \in \Gamma_{2}, \\ \eta(t, x)=0 & t>0 x \in \Gamma_{1}, \\ \eta(0, x)=\eta_{0}(x) & x \in \Omega .\end{cases}
$$

We assume that the output feedback control law

$$
u_{1}(t, x)=\left.\frac{\partial v_{1}(t, x)}{\partial \nu}\right|_{\Gamma_{3}}, \quad u_{2}(t, x)=0 .
$$

Then, system (46) becomes

$$
\begin{cases}\partial_{t} \eta(t, x)-\alpha \Delta \eta(t, x)+B . \nabla \eta(t, x)=0 & t>0 x \in \Omega, \\ \frac{\partial \eta(t, x)}{\partial \nu}=\mathcal{M} \eta(t, x) & t>0 x \in \Gamma_{4}, \\ \frac{\partial \eta(t, x)}{\partial \nu}=\left(0, \frac{\partial v_{2}(t, x)}{\partial \nu}\right) & t>0 x \in \Gamma_{3}, \\ \frac{\partial \eta(t, x)}{\partial \nu}=0 & t>0 x \in \Gamma_{2}, \\ \eta(t, x)=0 & t>0 x \in \Gamma_{1}, \\ \eta(0, x)=\eta_{0}(x) & x \in \Omega .\end{cases}
$$

Now, we state the well posedness and boundeness of the solution to system (44).

Lemma 10. Suppose that $r \in W^{1, \infty}\left(0, \infty, \mathcal{H}_{\Gamma_{3}}\right), v_{0} \in \mathbf{X}$, then, system (44) admits a unique solution $v \in C(0, \infty ; \mathbf{X})$ which is uniformly bounded for all $t \geqslant 0$, i.e. $\sup _{t \geqslant 0}\|v(t)\|_{\mathbf{X}}<+\infty$. Moreover, for $r \in H^{1}\left(0, \infty, \mathcal{H}_{s \Gamma_{3}}\right)$ we have $\lim _{t \rightarrow \infty}\|v(t)\|_{\mathbf{X}}=$ 0 .

Proof. In order to give the well posedness we separate the servo system (44) into tow subsystem $\rho_{1}$ and $\rho_{2}$ described respectively by

$$
\begin{cases}\partial_{t} \rho_{1}(t, x)-\alpha \Delta \rho_{1}(t, x)+B . \nabla \rho_{1}(t, x)=0 & t>0 x \in \Omega, \\ \frac{\partial \rho_{1}(t, x)}{\partial \nu}=\mathcal{M} \rho_{1}(t, x) & t>0 x \in \Gamma_{4}, \\ \rho_{1}(t, x)=r(t, x) & t>0 x \in \Gamma_{3}, \\ \frac{\partial \rho_{1}(t, x)}{\partial \nu}=0 & t>0 x \in \Gamma_{2}, \\ \rho_{1}(t, x)=0 & t>0 x \in \Gamma_{1}, \\ \rho_{1}(0, x)=\rho_{10}(x) & x \in \Omega,\end{cases}
$$


and

$$
\begin{cases}\partial_{t} \rho_{2}(t, x)-\alpha \Delta \rho_{2}(t, x)+B . \nabla \rho_{2}(t, x)=0 & t>0 x \in \Omega, \\ \frac{\partial \rho_{2}(t, x)}{\partial \nu}=\mathcal{M} \rho_{2}(t, x) & t>0 x \in \Gamma_{4}, \\ \rho_{2}(t, x)=0 & t>0 x \in \Gamma_{3}, \\ \frac{\partial \rho_{2}(t, x)}{\partial \nu}=0 & t>0 x \in \Gamma_{2}, \\ \rho_{2}(t, x)=y_{m}(t) & t>0 x \in \Gamma_{1}, \\ \rho_{2}(0, x)=\rho_{20}(x) & x \in \Omega,\end{cases}
$$

First,we introduce the Dirichlet map $\Lambda_{1} \in \mathcal{L}\left(\mathcal{H}_{\Gamma_{3}} ; \mathcal{H}_{s}^{\frac{1}{2}}\right)$ see [18, pages 188]

$$
\begin{cases}-\alpha \Delta z+B . \nabla z=0 & t>0, x \in \Omega, \\ \frac{\partial z}{\partial \nu}=\mathcal{M} z & t>0, x \in \Gamma_{4}, \\ z=r(t, x) & t>0, x \in \Gamma_{3} \\ \frac{\partial z}{\partial \nu}=0 & t>0, x \in \Gamma_{2} \\ z=0 & t>0, x \in \Gamma_{1}\end{cases}
$$

for $r \in W^{1, \infty}\left(0, \infty ; \mathcal{H}_{\Gamma_{3}}\right)$ then $z \in W^{1, \infty}\left(0, \infty ; \mathcal{H}_{s}^{\frac{1}{2}}\right)$. Moreover, we have

$$
\|z(t)\|_{\mathcal{H}_{s}^{\frac{1}{2}}} \leqslant C_{1}\|r(t)\|_{\mathcal{H}_{\Gamma_{3}}}\left\|z_{t}(t)\right\|_{\mathcal{H}_{s}^{\frac{1}{2}}} \leqslant C_{2}\left\|r_{t}(t)\right\|_{\mathcal{H}_{\Gamma_{3}}}, t \geqslant 0 .
$$

From the Sobolev embedding theorem, it follows that

$$
\left\|z_{t}(t)\right\|_{\mathbf{X}} \leqslant C_{2}\|r(t)\|_{\mathbf{X}}, t \geqslant 0 .
$$

Now, by the Dirichlet map $\Lambda_{1}$ we introduce $\bar{v}_{1}(t, x)=\rho_{1}(t, x)-z(t, x)$, then $\bar{v}_{1}$ satisfies

$$
\begin{cases}\partial_{t} \bar{v}_{1}(t, x)-\alpha \Delta \bar{v}_{1}(t, x)+B . \nabla \bar{v}_{1}(t, x)=-z_{t}(t, x) & t>0 x \in \Omega, \\ \frac{\partial \bar{v}_{1}(t, x)}{\partial \nu}=\mathcal{M} \bar{v}_{1}(t, x) & t>0 x \in \Gamma_{4}, \\ \bar{v}_{1}(t, x)=0 & t>0 x \in \Gamma_{3}, \\ \frac{\partial \bar{v}_{1}(t, x)}{\partial \nu}=0 & t>0 x \in \Gamma_{2}, \\ \bar{v}_{1}(t, x)=0 & t>0 x \in \Gamma_{1}, \\ \bar{v}_{1}(0, x)=\bar{v}_{01}(x) & x \in \Omega,\end{cases}
$$

can be written as

$$
\partial_{t} \bar{v}_{1}(t, .)=\overline{\mathcal{A}} \bar{v}_{1}(t, .)+B_{1} z_{t}(t, .),
$$

where $B_{1}=-I$ and the operator $\overline{\mathcal{A}}$ is given by

$$
\overline{\mathcal{A}}=\Delta \phi+B . \nabla \phi, \forall \phi \in D(\overline{\mathcal{A}}),
$$

and

$$
D(\overline{\mathcal{A}})=\left\{\phi \in \mathbf{Z} ;\left.\frac{\partial \phi}{\partial \nu}\right|_{\Gamma_{4}}=\mathcal{M} \phi ;\left.\frac{\partial \phi}{\partial \nu}\right|_{\Gamma_{2}}=0 ;\left.\phi\right|_{\Gamma_{1}}=\left.\phi\right|_{\Gamma_{3}}=0\right\} .
$$


It is easy to show that $\overline{\mathcal{A}}$ generates an exponentially stable $C_{0}$-semigroup $e^{\overline{\mathcal{A}}}$. Then using (51) and (52) it follows that the system (49) has a unique solution $\rho_{1} \in C(0, \infty ; \mathbf{X})$. Moreover, using [26, Lemma 1.1], we conclude that

$$
\lim _{t \rightarrow \infty}\left\|\rho_{1}(t)\right\| \mathbf{x}=0
$$

By the same argument we introduce $\Lambda_{2}: \mathcal{L}\left(\mathcal{H}_{s \Gamma_{1}} ; \mathcal{H}_{s}^{\frac{1}{2}}\right)$. Then

$$
\begin{cases}-\alpha \Delta y+B . \nabla y=0 & x \in \Omega, \\ \frac{\partial y}{\partial \nu}=\mathcal{M} . y & x \in \Gamma_{4}, \\ y=0 & x \in \Gamma_{3}, \\ \frac{\partial y}{\partial \nu}=0 & x \in \Gamma_{2}, \\ y=y_{m}(t) & x \in \Gamma_{1},\end{cases}
$$

with $y_{m} \in W^{1, \infty}\left(0, \infty ; \mathcal{H}_{s \Gamma_{1}}\right)$, then $y \in W^{1, \infty}\left(0, \infty ; \mathcal{H}_{s}^{\frac{1}{2}}\right)$. Finally, the system (50) has a unique solution, $\rho_{2} \in C(0, \infty ; \mathbf{X})$ and by [26, Lemma 1.1], we have

$$
\lim _{t \rightarrow \infty}\left\|\rho_{2}(t)\right\|_{\mathbf{x}}=0
$$

From (53) and (55), we have

$$
\lim _{t \rightarrow \infty}\|v(t)\|_{\mathbf{X}}=0
$$

This finish the proof.

Lemma 11. Let us assume $\eta_{0} \in \mathbf{X}$, the system (39) admits a unique solution $\eta \in C(0, \infty ; \mathbf{X})$ such that

$$
\|\eta(t)\| \mathbf{x} \leqslant e^{-t}\left\|\eta_{0}\right\|_{\mathbf{X}}+C\|v(t)\|_{\mathbf{x}}, \quad \forall t>0 .
$$

for a constant $C>0$. Moreover, for any $\tau>0$ there exist $M_{0}>0$ depending on $\tau$ and $\delta_{0}>0$ such that

$(57)$

$$
\left\|\frac{\partial \eta}{\partial \nu}\right\|_{\mathcal{H}_{\Gamma_{1}}} \leqslant M_{0} e^{-\delta_{0} t}\left\|\eta_{0}\right\|_{\mathbf{X}}+C_{1}\|v(t)\|_{\mathbf{X}}, \quad\|\eta\|_{\mathcal{H}_{\Gamma_{3}}} \leqslant M_{0} e^{-\delta_{0} t}\left\|\eta_{0}\right\|_{\mathbf{X}}+C_{1}\|v(t)\|_{\mathbf{X}},
$$

for all $t>\tau$ and for a positive constant $C_{1}$.

Proof. The proof is similar to the proof of Lemmas 9 and 10

Now, we are able to give an asymptotical estimation of the disturbance $d(t)$. From the error system it follows

$$
\frac{\partial \tilde{w}}{\partial \nu}(t, x)=\frac{\partial \hat{w}}{\partial \nu}(t, x)-\frac{\partial w}{\partial \nu}(t, x)=\frac{\partial \hat{w}}{\partial \nu}(t, x)-d(t) \quad \forall t \geqslant 0, x \in \Gamma_{1} .
$$

Consequently, from (40) and (57) then it follows thus the disturbance can be estimated by

$$
d \approx \frac{\partial \hat{w}}{\partial \nu}, \text { for a large value of } t
$$


6.3. Control design and closed-loop system. Now, we analyze the performance output tracking to the closed loop system of (37). Using the feedback law (47), the closed loop is given by

$$
\begin{cases}\partial_{t} w(t, x)-\alpha \Delta w(t, x)+B . \nabla w(t, x)=0 & t>0 x \in \Omega, \\ \frac{\partial w(t, x)}{\partial \nu}=\mathcal{M} w(t, x) & t>0 x \in \Gamma_{4}, \\ \frac{\partial w(t, x)}{\partial \nu}=\left(\frac{\partial v_{1}(t, x)}{\partial \nu}, 0\right) & t>0 x \in \Gamma_{3}, \\ \frac{\partial w(t, x)}{\partial \nu}=0 & t>0 x \in \Gamma_{2}, \\ \frac{\partial w(t, x)}{\partial \nu}=d(t) & t>0 x \in \Gamma_{1}, \\ \partial \hat{t}(t, x)-\alpha \Delta \hat{w}(t, x)+B . \nabla \hat{w}(t, x)=0 & t>0 x \in \Omega, \\ \frac{\partial \hat{w}(t, x)}{\partial \nu}=\mathcal{M} \hat{w}(t, x) & t>0 x \in \Gamma_{4}, \\ \frac{\partial \hat{w}(t, x)}{\partial \nu}=\left(\frac{\partial v_{1}(t, x)}{\partial \nu}, 0\right) & t>0 x \in \Gamma_{3}, \\ \frac{\partial \hat{w}(t, x)}{\partial \nu}=0 & t>0 x \in \Gamma_{2}, \\ \hat{w}(t, x)=y_{m}(t, x) & t>0 x \in \Gamma_{1}, \\ \partial t(t, x)-\alpha \Delta v(t, x)+B . \nabla v(t, x)=0 & t>0 x \in \Omega_{1}, \\ \frac{\partial v(t, x)}{\partial \nu}=\mathcal{M} v(t, x) & t>0 x \in \Gamma_{4}, \\ v(t, x)=r(t) & t>0 x \in \Gamma_{3}, \\ \frac{\partial v(t, x)}{\partial \nu}=0 & t>0 x \in \Gamma_{2}, \\ v(t, x)=\hat{w}(t, x) & t>0 x \in \Gamma_{1}, \\ w(0, x)=w_{0}(x) & x \in \Omega, \\ \hat{w}(0, x)=\hat{w}_{0}(x) & x \in \Omega, \\ v(0, x)=v_{0}(x) & x \in \Omega\end{cases}
$$

Theorem 12. Let $r \in W^{1, \infty}\left(0, \infty, \mathcal{H}_{\Gamma_{3}}\right)$, for any initial value $\left(w_{0}, \hat{w}_{0}, v_{0}\right) \in \mathbf{X}^{3}$, the closed-loop system (59) admits a unique solution $(w, \hat{w}, v) \in C\left(0 ; \infty ; \mathbf{X}^{3}\right)$ such that

1.

$$
\sup _{t \geqslant 0}\left(\|\hat{w}(t)\|_{\mathbf{x}}+\|w(t)\|_{\mathbf{X}}+\|v(t)\|_{\mathbf{x}}\right)<+\infty, \quad \forall t \geqslant 0
$$

2. There exists a constant $M$ depending on the initial data $\left(w_{0}, \hat{w}_{0}\right)$ and $\mu>0$ such that

$$
\|w(t)-\hat{w}(t)\|_{\mathbf{x}} \leq M e^{-\mu t}, \quad \forall t \geqslant 0
$$

and for any $\tau>0$ we have

$$
\|e(t)\|_{\mathbf{x}} \leq M_{1} e^{-\mu_{1} t}, \quad \forall t \geqslant 0, \quad \forall t \geqslant 0,
$$

where $M_{1}$ depending on the initial data $\left(w_{0}, \hat{w}_{0}, v_{0}\right)$ and a constant $\mu_{1}>0$.

3. Moreover, for $r \in H^{1}\left(0, \infty, \mathcal{H}_{\Gamma_{3}}\right)$ we have the asymptotically stability of system (59)

$$
\lim _{t \rightarrow \infty}\left(\|\hat{w}(t)\| \mathbf{x}+\|w(t)\|_{\mathbf{x}}+\|v(t)\|_{\mathbf{x}}\right)=0
$$


Proof. Let $\tilde{w}(t, x)=\hat{w}(t, x)-w(t, x)$ and $\eta(t, x)=v(t, x)-\hat{w}(t, x)$, then the closed loop system (59) is equivalent to

$(61)$

$$
\begin{cases}\partial_{t} \tilde{w}(t, x)-\alpha \Delta \tilde{w}(t, x)+B . \nabla \tilde{w}(t, x)=0 & t>0 x \in \Omega, \\ \frac{\partial \tilde{w}(t, x)}{\partial \nu}=\mathcal{M} \tilde{w}(t, x) & t>0 x \in \Gamma_{4}, \\ \frac{\partial \tilde{w}(t, x)}{\partial \nu}(t, x)=0 & t>0 x \in \Gamma_{3}, \\ \frac{\partial \tilde{w}(t, x)}{\partial \nu}=0 & t>0 x \in \Gamma_{2}, \\ \tilde{w}(t, x)=0 & t>0 x \in \Gamma_{1}, \\ \partial_{t} \eta(t, x)-\alpha \Delta \eta(t, x)+B . \nabla \eta(t, x)=0 & t>0 x \in \Omega, \\ \frac{\partial \eta(t, x)}{\partial \nu}=\mathcal{M} \eta(t, x) & t>0 x \in \Gamma_{4}, \\ \frac{\partial \eta(t, x)}{\partial \nu}(t, x)=\left(0, \frac{\partial v_{2}(t, x)}{\partial \nu}\right) & t>0 x \in \Gamma_{3}, \\ \frac{\partial \eta(t, x)}{\partial \nu}=0 & t>0 x \in \Gamma_{2}, \\ \eta(t, x)=0 & t>0 x \in \Gamma_{1}, \\ \partial_{t} \rho_{2}(t, x)-\alpha \Delta \rho_{2}(t, x)+B . \nabla \rho_{2}(t, x)=0 & t>0 x \in \Omega, \\ \frac{\partial \rho_{2}(t, x)}{\partial \nu}=\mathcal{M} \rho_{2}(t, x) & t>0 x \in \Gamma_{4}, \\ \rho_{2}(t, x)=0 & t>0 x \in \Gamma_{3}, \\ \frac{\partial \rho_{2}(t, x)}{\partial \nu}=0 & t>0 x \in \Gamma_{2}, \\ \rho_{2}(t, x)=0 & t>0 x \in \Gamma_{1}, \\ \tilde{w}(0, x)=\tilde{w}_{0}(x) & x \in \Omega, \\ \eta(0, x)=\eta_{0}(x) & x \in \Omega, \\ \rho_{2}(0, x)=\rho_{20}(x) & x \in \Omega,\end{cases}
$$

and

$$
\begin{cases}\partial_{t} \rho_{1}(t, x)-\alpha \Delta \rho_{1}(t, x)+B . \nabla \rho_{1}(t, x)=0 & t>0 x \in \Omega, \\ \frac{\partial \rho_{1}(t, x)}{\partial \nu}=\mathcal{M} \rho_{1}(t, x) & t>0 x \in \Gamma_{4}, \\ \rho_{1}(t, x)=r(t, x) & t>0 x \in \Gamma_{3}, \\ \frac{\partial \rho_{1}(t, x)}{\partial \nu}=0 & t>0 x \in \Gamma_{2}, \\ \rho_{1}(t, x)=y_{m}(t, x) & t>0 x \in \Gamma_{1}, \\ \rho_{1}(0, x)=\rho_{10}(x) & x \in \Omega\end{cases}
$$


Thus system (61) becomes

$$
\begin{cases}\partial_{t} \eta(t, x)-\alpha \Delta \eta(t, x)+B . \nabla \eta(t, x)=0 & t>0 x \in \Omega, \\ \frac{\partial \eta(t, x)}{\partial \nu}=\mathcal{M} \eta(t, x) & t>0 x \in \Gamma_{4}, \\ \frac{\partial \eta(t, x)}{\partial \nu}(t, x)=\left(0, \frac{\partial \rho_{12}(t, x)}{\partial \nu}+\frac{\partial \rho_{22}(t, x)}{\partial \nu}\right) & t>0 x \in \Gamma_{3}, \\ \frac{\partial \eta(t, x)}{\partial \nu}=0 & t>0 x \in \Gamma_{2}, \\ \eta(t, x)=0 & t>0 x \in \Gamma_{1}, \\ \partial \rho_{2}(t, x)-\alpha \Delta \rho_{2}(t, x)+B . \nabla \rho_{2}(t, x)=0 & t>0 x \in \Omega, \\ \frac{\partial \rho_{2}(t, x)}{\partial \nu}=\mathcal{M} \rho_{2}(t, x) & t>0 x \in \Gamma_{4}, \\ \rho_{2}(t, x)=0 & t>0 x \in \Gamma_{3}, \\ \frac{\partial \rho_{2}(t, x)}{\partial \nu}=0 & t>0 x \in \Gamma_{2}, \\ \rho_{2}(t, x)=0 & t>0 x \in \Gamma_{1}, \\ \eta(0, x)=\eta_{0}(x) & x \in \Omega, \\ \rho_{2}(0, x)=\rho_{20}(x) & x \in \Omega .\end{cases}
$$

System (66) can be rewritten as follows

$$
\frac{d}{d t}\left(\eta(t, .), \rho_{2}(t, .)\right)=\mathbb{A}\left(\eta(t, .), \rho_{2}(t, .)\right)+\mathbb{B} \frac{\partial \rho_{1}(t, .)}{\partial \nu}
$$

Now, we need to show that $\mathbb{B} v_{1}=\left(0,\left.\frac{\partial v_{12}(t, .)}{\partial \nu}\right|_{\Gamma_{1}}\right)$, for all $v=v_{1}+v_{2} \in \overline{\mathcal{A}}$, is an admissible operator for $e^{\overline{\mathcal{A}} t}$.

$$
\mathbb{A}=(\alpha \Delta \phi-B . \nabla \phi, \alpha \Delta \psi-B . \nabla \psi), \forall(\phi, \psi) \in D(\mathbb{A}),
$$

and

(64)

$$
\begin{aligned}
& D(\mathbb{A})=\left\{(\phi, \psi) \in \mathbf{Z} ;\left.\frac{\partial \phi}{\partial \nu}\right|_{\Gamma_{4}}=\mathcal{M} \phi ;\left.\frac{\partial \phi}{\partial \nu}\right|_{\Gamma_{2}}=0 ;\left.\phi\right|_{\Gamma_{1}}=0 ;\right. \\
& \left.\frac{\partial \phi(t, x)}{\partial \nu} \Gamma_{\Gamma_{3}}=\left.\left(0, \frac{\partial \psi_{2}(t, x)}{\partial \nu}\right)_{\Gamma_{3}} \frac{\partial \psi}{\partial \nu}\right|_{\Gamma_{4}}=\mathcal{M} \psi ;\left.\frac{\partial \psi}{\partial \nu}\right|_{\Gamma_{2}}=0 ;\left.\psi\right|_{\Gamma_{1}}=\left.\psi\right|_{\Gamma_{3}}=0\right\} .
\end{aligned}
$$

Let

$$
\mathbb{A}^{*}=(\alpha \Delta \eta+\nabla \cdot(B \phi), \alpha \Delta \psi+\nabla \cdot(B \psi)), \forall(\phi, \psi) \in D\left(\mathbb{A}^{*}\right)
$$

$$
\begin{gathered}
D\left(\mathbb{A}^{*}\right)=\left\{(\phi, \psi) \in \mathbf{Z} ;\left.\frac{\partial \phi}{\partial \nu}\right|_{\Gamma_{4}}=\mathcal{M}^{*} \phi ;\left.\frac{\partial \phi}{\partial \nu}\right|_{\Gamma_{2}}=0 ;\left.\phi\right|_{\Gamma_{1}}=0 ; \phi_{\Gamma_{\Gamma_{3}}}=\left(0,\left.\psi_{2}\right|_{\Gamma_{3}}\right)\right. \\
\left.\left.\frac{\partial \psi}{\partial \nu}\right|_{\Gamma_{4}}=\mathcal{M}^{*} \psi ;\left.\frac{\partial \psi}{\partial \nu}\right|_{\Gamma_{2}}=0 ;\left.\psi\right|_{\Gamma_{1}}=\left.\psi\right|_{\Gamma_{3}}=0\right\} . \\
\mathcal{M}^{*}=\left[\begin{array}{ll}
\gamma_{f} & -\gamma_{p} \\
\gamma_{f} & -\gamma_{p}
\end{array}\right], \mathbb{B} \text { is an observation operator for the adjoint semigroup } e^{\mathbb{A}^{*} t} .
\end{gathered}
$$

So we use to fact that $\mathbb{B}^{*} \mathbb{A}^{*}$ is bounded from $\mathbf{Z}$ to $\mathcal{H}_{\Gamma_{1}}^{2}$. The adjoint problem is given 
by

$$
\begin{cases}\partial_{t} \eta^{*}(t, x)-\alpha \Delta \eta^{*}(t, x)-\nabla \cdot\left(B \eta^{*}(t, x)\right)=0 & t>0 x \in \Omega, \\ \frac{\partial \eta^{*}(t, x)}{\partial \nu}=\mathcal{M}^{*} \eta^{*}(t, x) & t>0 x \in \Gamma_{4}, \\ \eta^{*}(t, x)=\left(0, \rho_{22}^{*}(t, x)\right) & t>0 x \in \Gamma_{3}, \\ \frac{\partial \eta^{*}(t, x)}{\partial \nu}=0 & t>0 x \in \Gamma_{2}, \\ \eta^{*}(t, x)=0 & t>0 x \in \Gamma_{1}, \\ \partial t \rho_{2}^{*}(t, x)-\alpha \Delta \rho_{2}^{*}(t, x)-\nabla \cdot\left(B \rho^{*}(t, x)\right)=0 & t>0 x \in \Omega, \\ \frac{\partial \rho_{2}^{*}(t, x)}{\partial \nu}=\mathcal{M}^{*} \rho_{2}^{*}(t, x) & t>0 x \in \Gamma_{4}, \\ \rho_{2}^{*}(t, x)=0 & t>0 x \in \Gamma_{3}, \\ \frac{\partial \rho_{2}^{*}(t, x)}{\partial \nu}=0 & t>0 x \in \Gamma_{2}, \\ \rho_{2}^{*}(t, x)=0 & t>0 x \in \Gamma_{1}, \\ \eta^{*}(0, x)=\eta_{0}^{*}(x) & x \in \Omega, \\ \rho_{2}^{*}(0, x)=\rho_{20}^{*}(x) & x \in \Omega .\end{cases}
$$

We need to show that for every $\tau^{*}$ there exists $C=C_{\tau^{*}}$ such that

$$
\int_{0}^{\tau^{*}} \int_{\Gamma_{1}} \rho_{2}^{* 2}(t, x) d t d x \leqslant C\left(\left\|\rho_{20}^{*}\right\|_{\mathbf{X}}^{2}+\left\|\eta_{0}^{*}\right\|_{\mathbf{X}}^{2}\right)
$$

By the Sobolev embedding theorem and the trace theorem, we deduce that $\mathbb{B}$ is admissible for $e^{\mathbb{A} t}$.

From Lemmas 9 and 11 for any $\tau>0$, there exist $M_{1}$ and $M_{2}$ depending on $\tilde{w}_{01}$ and $\mu_{0}$ and $\mu_{1}, \mu_{2}>0$

$$
\begin{gathered}
\|\tilde{w}(t)\|_{\mathbf{x}}+\|\eta(t)\|_{\mathbf{X}} \leqslant M_{1} e^{-\mu_{1} t}, t \geqslant 0, \\
\|\tilde{w}(t)\|_{\mathcal{H}_{\Gamma_{3}}}+\|\eta(t)\|_{\mathcal{H}_{\Gamma_{3}}} \leqslant M_{2} e^{-\mu_{2} t}, t \geqslant \tau .
\end{gathered}
$$

The $(w, \hat{w}, v)$-system is well-posed and admits a unique solution. From Lemma 9, (67) and (68) we conclude claim 1. Moreover, claim 2 follow from (67) and (68). So, we have $e(t, x)=y_{0}(t, x)-r(t, x)=-\left.\tilde{w}(t, x)\right|_{\Gamma_{3}}-\left.\eta(t, x)\right|_{\Gamma_{3}}$, then the claim 3 follows from (68). Finally, by Lemma 10 and (67), estimate (60) holds as well.

Now, we discuss the the performance of our tracking problem for some noise measurement $\sigma$.

Proposition 13. Suppose that $r \in H^{1}\left(0, \infty, \mathcal{H}_{\Gamma_{3}}\right)$, assuming $y_{m}=w(t, x)_{\left.\right|_{\Gamma_{1}}}+$ $\sigma(t)$ where $\sigma$ is the noise $\sigma \in W^{2, \infty}\left(0, \infty, \mathcal{H}_{\Gamma_{1}}\right)$. Then, for $\left(w_{0}, \hat{w}_{0}, v_{0}\right)$, the closed-loop system (59) admits a unique solution $(w, \hat{w}, v) \in C\left(0, \infty ; \mathbf{X}^{3}\right)$. The output tracking is robust with respect to $\sigma$. In addition, for any fixed $\tau>0$, there exists two constants $M>0$ which depend only on initial value and a constant $\mu>0$ such that

$$
\|e(t)\|_{\mathbf{x}} \leq M\left(e^{-\mu t}+\|\sigma\|_{W^{2, \infty}\left(0, \infty, \mathcal{H}_{\Gamma_{1}}\right)}\right), \quad \forall t \geqslant 0 .
$$


Proof. Let $\tilde{w}(t, x)=\hat{w}(t, x)-w(t, x)$ then the error of of estimation is governed by

$$
\begin{cases}\partial_{t} \tilde{w}(t, x)-\alpha \Delta \tilde{w}(t, x)+B . \nabla \tilde{w}(t, x)=0 & t>0 x \in \Omega, \\ \frac{\partial \tilde{w}(t, x)}{\partial \nu}=\mathcal{M} \tilde{w}(t, x) & t>0 x \in \Gamma_{4}, \\ \frac{\partial \tilde{w}(t, x)}{\partial \nu}(t, x)=0 & t>0 x \in \Gamma_{3}, \\ \frac{\partial \tilde{w}(t, x)}{\partial \nu}=0 & t>0 x \in \Gamma_{2}, \\ \tilde{w}(t, x)=\sigma(t, x) & t>0 x \in \Gamma_{1}, \\ \tilde{w}(0, x)=\tilde{w}_{0}(x) & x \in \Omega .\end{cases}
$$

Now, in order to show the well posedness of $(\tilde{w}, \eta, v)$-well process as previously by introducing the Dirichlet map $\Lambda_{3} \in \mathcal{L}\left(\mathcal{H}_{\Gamma_{3}} ; \mathcal{H}_{s}^{\frac{1}{2}}\right), \Lambda_{3}(\sigma)=z$ where is the solution of

$$
\begin{cases}-\alpha \Delta z+B \cdot \nabla z=0 & x \in \Omega \\ \frac{\partial z}{\partial \nu}=\mathcal{M} . z & x \in \Gamma_{4} \\ \frac{\partial z}{\partial \nu}=0 & x \in \Gamma_{3} \\ \frac{\partial z}{\partial \nu}=0 & x \in \Gamma_{2} \\ z=\sigma & x \in \Gamma_{1}\end{cases}
$$

we have $\sigma \in W^{2, \infty}\left(0, \infty ; \mathcal{H}_{\Gamma_{1}}\right)$ then it follows that $z \in W^{2, \infty}\left(0, \infty ; \mathcal{H}_{s}^{1 / 2}\right)$ and for all $t \geqslant 0$ we have

$$
\|z(t)\|_{\mathcal{H}_{s}^{1 / 2}} \leqslant C_{1}\|\sigma(t)\|_{\mathcal{H}_{\Gamma_{1}}}, \quad\left\|z_{t}(t)\right\|_{\mathcal{H}_{s}^{1 / 2}} \leqslant C_{2}\left\|\sigma_{t}(t)\right\|_{\mathcal{H}_{\Gamma_{1}}} .
$$

Then, we can deduce there exist $C>0$

$$
\left\|z_{t}(t)\right\|_{L^{2}(\Omega)} \leqslant C\|\sigma\|_{W^{2, \infty}\left(0, \infty ; \mathcal{H}_{\Gamma_{1}}\right)}, \quad \forall t \geqslant 0 .
$$

Thus, using the Dirichlet map $\Lambda_{3}$ and introducing the new variable $\bar{w}(t, x)=\tilde{w}(t, x)-$ $z(t, x)$, system (69) becomes

$$
\begin{cases}\partial_{t} \bar{w}(t, x)-\alpha \Delta \bar{w}(t, x)+B . \nabla \bar{w}(t, x)=-\partial_{t} z & t>0 x \in \Omega, \\ \frac{\partial \bar{w}(t, x)}{\partial \nu}=\mathcal{M} \bar{w}(t, x) & t>0 x \in \Gamma_{4}, \\ \frac{\partial \bar{w}(t, x)}{\partial \nu}(t, x)=0 & t>0 x \in \Gamma_{3}, \\ \frac{\partial \bar{w}(t, x)}{\partial \nu}=0 & t>0 x \in \Gamma_{2}, \\ \bar{w}(t, x)=0 & t>0 x \in \Gamma_{1}, \\ \bar{w}(0, x)=\bar{w}_{0}(x) & x \in \Omega .\end{cases}
$$

We know that $\tilde{\mathcal{A}}$ defined in the proof of Lemma 9 generates our analytic semigroup $\tilde{S}(t)$, thus $\bar{w}_{0} \in \mathbf{X}$, the mild solution of (69) given by

$$
\bar{w}(t, x)=S(t) \bar{w}_{0}-\int_{0}^{t} S(t-s) z_{s}(s, .) d s .
$$


Using the parabolic regularity and then nonhomogenous parabolic equation see [25], we can deduce

$$
\|\bar{w}(t)\|_{\mathbf{Z}} \leqslant C\left(e^{-\mu_{0} t}\left\|\bar{w}_{0}\right\|_{L^{2}(\Omega)}+\|\sigma\|_{W^{2, \infty}\left(0, \infty ; \mathcal{H}_{\Gamma_{1}}\right)}\right), \quad \forall t \geqslant 0
$$

where $C>0$, then by trace theorem, it follows that

$$
\|\bar{w}(t)\|_{\mathcal{H}_{\Gamma_{1}}} \leqslant C_{1}\left(e^{-\mu_{0} t}\left\|\bar{w}_{0}\right\|_{L^{2}(\Omega)}+\|\sigma\|_{W^{2, \infty}\left(0, \infty ; \mathcal{H}_{\Gamma_{1}}\right)}\right), \quad \forall t \geqslant 0,
$$

Thus finished the proof.

7. Conclusion and comments. In this paper, a mathematical analysis of a system of two dimensional advection diffusion equations coupled at the boundary has been provided. This system of equations models the heat transfer in direct contact membrane distillation process. A new formulation of the problem based on semi group framework is introduced. Moreover, the well-posedness criteria for the system is provided using the operator theory. The co-current case has been also analyzed where it has been shown that the operator is diagonalizable. However, based the ADRC, we designed a feedback law for the DCMD system. The performance output exponentially tracks the reference signal is also showed. Moreover, we discussed the the robustness to the measurement noise. Finally, investigating the ADRC for this kind of parabolic system in order to track the membrane temperature it will be a difficult and interesting open question.

\section{REFERENCES}

[1] A. Alsaadi, L. Francis, G. Amy, and N. Ghaffour, Experimental and theoretical analyses of temperature polarization effect in vacuum membrane distillation, Journal of Membrane Science, 471 (2014), pp. 138-148, https://doi.org/10.1016/j.memsci.2014.08.005.

[2] H. Brezis, Functional analysis, Sobolev spaces and partial differential equations, Universitext, Springer, New York, 2011.

[3] T. Cieślak and Philippe Laurengt, Finite time blow-up for a one-dimensional quasilinear parabolic-parabolic chemotaxis system, Annales de l'Institut Henri Poincaré (C) Non Linear Analysis, 27 (2010), pp. 437 - 446, https://doi.org/10.1016/j.anihpc.2009.11.016.

[4] L. Corrias, B. Perthame, And H. ZaAg, Global solutions of some chemotaxis and angiogenesis systems in high space dimensions, Milan Journal of Mathematics, 72 (2004), pp. 1-28, https://doi.org/10.1007/s00032-003-0026-x.

[5] C. Desoer And C. A. Lin, Tracking and disturbance rejection of mimo nonlinear systems with pi controller, IEEE Transactions on Automatic Control, 30 (1985), pp. 861-867.

[6] M. El-Bourawi, Z. Ding, R. Ma, and M. Khayet, A framework for better understanding membrane distillation separation process, Journal of membrane science, 285 (2006), pp. 429, https://doi.org/10.1016/j.memsci.2006.08.002.

[7] F. Eleiwi, N. Ghaffour, A. S. Alsaadi, L. Francis, and T. Laleg-Kirati, Dynamic modeling and experimental validation for direct contact membrane distillation (DCMD) process, Desalination, 384 (2016), pp. 1-11.

[8] L. C. Evans, Partial Differential Equations, Graduate studies in mathematics, American Mathematical Society, second ed., 2010.

[9] M. Faierman, Regularity of solutions of an elliptic boundary value problem in a rectangle, Communications in Partial Differential Equations, 12 (1987), pp. 285-305, https://doi. org/10.1080/03605308708820493.

[10] H. FENG AND B. Z. GUO, A new active disturbance rejection control to output feedback stabilization for a one-dimensional anti-stable wave equation with disturbance, IEEE Transactions on Automatic Control, 62 (2017), pp. 3774-3787.

[11] B. A. Francis, The linear multivariable regulator problem, SIAM Journal on Control and Optimization, 15 (1977), pp. 486-505.

[12] W. Guo And B. Z. Guo, Performance output tracking for a wave equation subject to unmatched general boundary harmonic disturbance, Automatica, 68 (2016), pp. 194-202. 
[13] W. Guo, Z. C. Shao, And M. Krstic, Adaptive rejection of harmonic disturbance anticollocated with control in 1d wave equation, Automatica, 79 (2017), pp. 17-26.

[14] F. F. JiN AND B. Z. GuO, Performance boundary output tracking for one-dimensional heat equation with boundary unmatched disturbance, Automatica, 96 (2018), pp. 1-10.

[15] A. M. Karam, A. S. Alsaadi, N. Ghaffour, and T. Laleg-Kirati, Analysis of direct contact membrane distillation based on a lumped-parameter dynamic predictive model, Desalination, 402 (2017), pp. 50-61.

[16] M. Khayet, Advanced Membrane Technology and Applications, John Wiley \& Sons, Inc., 2008, ch. Membrane Distillation, pp. 297-369.

[17] J.-G. Lee, A. S. Alsaadi, A. M. Karam, L. Francis, S. Soukane, and N. Ghaffour, Total water production capacity inversion phenomenon in multi-stage direct contact membrane distillation: A theoretical study, Journal of Membrane Science, 544 (2017), pp. 126-134, https://doi.org/10.1016/j.memsci.2017.09.020.

[18] J. L. Lions and E. Magenes, Non-homogeneous boundary value problems and applications, vol. 1, Springer Science \& Business Media, 2012.

[19] T. Meurer, Control of Higher-Dimensional PDEs: Flatness and Backstepping Designs, Springer Science \& Business Media, 2012.

[20] T. Meurer And A. KugI, Trajectory planning for boundary controlled parabolic pdes with varying parameters on higher-dimensional spatial domains, IEEE Transactions on Automatic Control, 54 (2009), pp. 1854-1868.

[21] N. Mizoguchi and P. Souplet, Nondegeneracy of blow-up points for the parabolic KellerSegel system, Annales de l'Institut Henri Poincaré (C) Non Linear Analysis, 31 (2014), pp. 851-875, https://doi.org/10.1016/j.anihpc.2013.07.007.

[22] G. Naidu, W. G. Shim, S. Jeong, Y. Choi, N. Ghaffour, and S. Vigneswaran, Transport phenomena and fouling in vacuum enhanced direct contact membrane distillation: Experimental and modelling, Separation and Purification Technology, 172 (2017), pp. 285-295, https://doi.org/10.1016/j.seppur.2016.08.024.

[23] A. PAZY, Semigroups of Linear Operators and Applications to Partial Differential Equations, Applied Mathematical Sciences, Springer New York, 2012, https://books.google.fr/books? id=DQvpBwAAQBAJ.

[24] W. G. Shim, K. He, S. Gray, And I. S. Moon, Solar energy assisted direct contact membrane distillation (DCMD) process for seawater desalination, Separation and Purification Technology, 143 (2015), pp. 94-104, https://doi.org/http://doi.org/10.1016/j.seppur.2015. 01.028.

[25] M. Tucsnak And G. Weiss, Observation and Control for Operator Semigroups, Birkhäuser Advanced Texts Basler Lehrbücher, Birkhäuser Basel, 2009, https://doi.org/10.1007/ 978-3-7643-8994-9.

[26] H.-C. Zhou And B.-Z. Guo, Performance output tracking for one-dimensional wave equation subject to unmatched general disturbance and non-collocated control, European Journal of Control, 39 (2018), pp. 39-52.

[27] H. C. Zhou, B. Z. GuO, AND S. H. XIANG, Performance output tracking for multi-dimensional heat equation subject to unmatched disturbance and non-collocated control, IEEE Transactions on Automatic Control, to appear. 